\title{
A Comparative Analyses of the Complete Mitochondrial Genomes of Fungal Endosymbionts in Sogatella furcifera, White-Backed Planthoppers
}

\author{
Nak Jung Choi $\mathbb{D}^{1}{ }^{1}$ Hong $\mathrm{Xi}^{2,3}$ and Jongsun Park $\mathbb{D}^{2,3}$ \\ ${ }^{1}$ Crop Foundation Division, National Institute of Crop Science, RDA, Wanju, Republic of Korea \\ ${ }^{2}$ InfoBoss Inc., 301 room, 670, Seolleung-ro, Gangnam-gu, Seoul, Republic of Korea \\ ${ }^{3}$ InfoBoss Research Center, 301 room, 670, Seolleung-ro, Gangnam-gu, Seoul, Republic of Korea \\ Correspondence should be addressed to Nak Jung Choi; njchoi@korea.kr and Jongsun Park; starflr@infoboss.co.kr
}

Received 29 December 2020; Revised 3 April 2021; Accepted 8 May 2021; Published 9 June 2021

Academic Editor: Ertugrul Filiz

Copyright ( $\odot 2021$ Nak Jung Choi et al. This is an open access article distributed under the Creative Commons Attribution License, which permits unrestricted use, distribution, and reproduction in any medium, provided the original work is properly cited.

\begin{abstract}
Sogatella furcifera Horvath, commonly known as the white-backed planthoppers (WBPH), is an important pest in East Asian rice fields. Fungal endosymbiosis is widespread among planthoppers in the infraorder Fulgoromorpha and suborder Auchenorrhyncha. We successfully obtained complete mitogenome of five WBPH fungal endosymbionts, belonging to the Ophiocordycipitaceae family, from next-generation sequencing (NGS) reads obtained from S. furcifera samples. These five mitogenomes range in length from $55,390 \mathrm{bp}$ to $55,406 \mathrm{bp}$, which is shorter than the mitogenome of the fungal endosymbiont found in Ricania speculum, black planthoppers. Twenty-eight protein-coding genes (PCGs), 12 tRNAs, and 2 rRNAs were found in the mitogenomes. Two single-nucleotide polymorphisms, two insertions, and three deletions were identified among the five mitogenomes, which were fewer in number than those of four species of Ophiocordycipitaceae, Ophiocordyceps sinensis, Hirsutella thompsonii, Hirsutella rhossiliensis, and Tolypocladium inflatum. Noticeably short lengths (up to $18 \mathrm{bp}$ ) of simple sequence repeats were identified in the five WBPH fungal endosymbiont mitogenomes. Phylogenetic analysis based on conserved PCGs across 25 Ophiocordycipitaceae mitogenomes revealed that the five mitogenomes were clustered with that of $R$. speculum, forming an independent clade. In addition to providing the full mitogenome sequences, obtaining complete mitogenomes of $\mathrm{WBPH}$ endosymbionts can provide insights into their phylogenetic positions without needing to isolate the mtDNA from the host. This advantage is of value to future studies involving fungal endosymbiont mitogenomes.
\end{abstract}

\section{Introduction}

Sogatella furcifera Horvath commonly known as the whitebacked planthopper (WBPH) is a planthopper belonging to the infraorder Fulgoromorpha [1] and suborder Auchenorrhyncha [2]. It has migrated to temperate climates from subtropical regions and become a major pest in rice fields across East Asia [3-6]. In particular, migration from China to Japan via Korean peninsula has highlighted the extent of its spread across the region [7]. Sogatella furcifera has already been registered in the National Species List of Korea [8] indicating that this species has been frequently found within the country. It damages rice plants by feeding directly on them, pro- ducing a characteristic symptom, hopper burn [9]. Because of the importance of WBPH as a threat to agriculture, the mitochondrial genome (mitogenome) as well as whole genome sequences of $S$. furcifera has been sequenced successfully $[10,11]$. The fundamental background of WBPH genomic research is, therefore, well established. For example, the complete genome sequence of the Cardinium bacterial endosymbiont of $S$. furcifera was also completed from the same raw reads generated by the whole genome project [12]. Another bacterial endosymbiont of WBPH, Wolbachia, which alters host reproductions by parthenogenesis, feminization, male-killing, and induction of cytoplasmic incompatibility in arthropods [13], also causes the cytoplasmic 


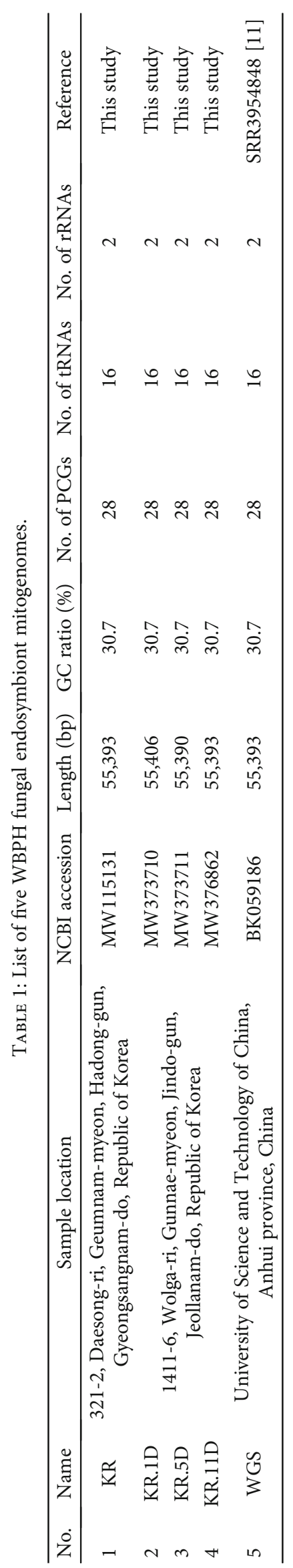


TABLE 2: List of genes in WBPH fungal endosymbiont mitogenomes.

\begin{tabular}{|c|c|c|c|c|c|c|c|}
\hline No. & Name & Type & Start position & End position & Length (bp) & Strand & No. of exons \\
\hline 1 & Cytochrome b & CDS & 160 & 8182 & 975 & Reverse & 5 \\
\hline 2 & LAGLIDADG/HNH endonuclease & CDS & 7429 & 7776 & 348 & Reverse & 1 \\
\hline 3 & NADH dehydrogenase subunit 5 & CDS & 8432 & 10,405 & 1974 & Reverse & 1 \\
\hline 4 & NADH dehydrogenase subunit $4 \mathrm{~L}$ & CDS & 10,405 & 10,680 & 276 & Reverse & 1 \\
\hline 5 & Cytochrome c oxidase subunit II & CDS & 10,896 & 13,952 & 894 & Reverse & 6 \\
\hline 6 & ATP synthase F0 subunit c & CDS & 14,052 & 14,237 & 186 & Reverse & 1 \\
\hline 7 & NADH dehydrogenase subunit 3 & CDS & 14,315 & 14,968 & 447 & Reverse & 2 \\
\hline 8 & LAGLIDADG endonuclease & CDS & 14,683 & 14,880 & 198 & Reverse & 1 \\
\hline 9 & NADH dehydrogenase subunit 2 & CDS & 14,969 & 18,193 & 1431 & Reverse & 2 \\
\hline 10 & Hypothetical protein & CDS & 15,626 & 16,390 & 765 & Reverse & 1 \\
\hline 11 & tRNA-Met & tRNA & 18,210 & 18,283 & 74 & Reverse & 1 \\
\hline 12 & tRNA-His & tRNA & 18,325 & 18,398 & 74 & Reverse & 1 \\
\hline 13 & tRNA-Leu & tRNA & 18,485 & 18,569 & 85 & Reverse & 1 \\
\hline 14 & tRNA-Lys & tRNA & 18,570 & 18,642 & 73 & Reverse & 1 \\
\hline 15 & tRNA-Phe & tRNA & 18,643 & 18,715 & 73 & Reverse & 1 \\
\hline 16 & tRNA-Leu & tRNA & 19,673 & 19,755 & 83 & Reverse & 1 \\
\hline 17 & tRNA-Met & tRNA & 19,758 & 19,830 & 73 & Reverse & 1 \\
\hline 18 & tRNA-Glu & tRNA & 19,904 & 19,976 & 73 & Reverse & 1 \\
\hline 19 & Large subunit rRNA & rRNA & 20,067 & 27,337 & 4793 & Reverse & 3 \\
\hline 20 & Ribosomal protein S3 & CDS & 20,672 & 21,916 & 1245 & Reverse & 1 \\
\hline 21 & tRNA-Pro & tRNA & 27,400 & 27,471 & 72 & Reverse & 1 \\
\hline 22 & NADH dehydrogenase subunit 6 & CDS & 27,677 & 28,441 & 765 & Reverse & 1 \\
\hline 23 & tRNA-Gly & tRNA & 28,544 & 28,614 & 71 & Reverse & 1 \\
\hline 24 & Cytochrome c oxidase subunit III & CDS & 29,224 & 32,387 & 795 & Reverse & 3 \\
\hline 25 & Intron-encoded endonuclease aI1 & CDS & 29,932 & 30,138 & 207 & Reverse & 1 \\
\hline 26 & Hypothetical protein & CDS & 30,395 & 30,664 & 270 & Reverse & 1 \\
\hline 27 & Alpha-beta-hydrolase & CDS & 30,423 & 30,686 & 264 & Forward & 1 \\
\hline 28 & tRNA-Asn & tRNA & 32,437 & 32,508 & 72 & Reverse & 1 \\
\hline 29 & Small subunit rRNA & rRNA & 34,254 & 35,615 & 1362 & Reverse & 1 \\
\hline 30 & ATP synthase F0 subunit a & CDS & 35,918 & 38,001 & 762 & Reverse & 2 \\
\hline 31 & ATP synthase F0 subunit 8 & CDS & 38,061 & 38,237 & 177 & Reverse & 1 \\
\hline 32 & NADH dehydrogenase subunit 4 & CDS & 38,620 & 40,035 & 1416 & Reverse & 1 \\
\hline 33 & NADH dehydrogenase subunit 1 & CDS & 40,119 & 42,582 & 1107 & Reverse & 4 \\
\hline 34 & tRNA-Arg & tRNA & 42,726 & 42,799 & 74 & Reverse & 1 \\
\hline 35 & Cytochrome $\mathrm{c}$ oxidase subunit I & CDS & 42,809 & 55,293 & 1314 & Reverse & 7 \\
\hline 36 & LAGLIDADG endonuclease & CDS & 43,660 & 44,880 & 1221 & Reverse & 1 \\
\hline 37 & LAGLIDADG endonuclease & CDS & 45,125 & 46,054 & 930 & Reverse & 1 \\
\hline 38 & LAGLIDADG endonuclease & CDS & 46,857 & 48,248 & 1392 & Reverse & 1 \\
\hline 39 & LAGLIDADG endonuclease & CDS & 48,620 & 49,483 & 864 & Reverse & 1 \\
\hline 40 & LAGLIDADG endonuclease & CDS & 49,525 & 50,814 & 1290 & Reverse & 1 \\
\hline 41 & LAGLIDADG endonuclease & CDS & 50,865 & 51,734 & 870 & Reverse & 1 \\
\hline 42 & Hypothetical protein & CDS & 54,290 & 54,850 & 561 & Forward & 1 \\
\hline
\end{tabular}

incompatibility in WBPH together with Cardinium endosymbiont [14].

Besides these bacterial endosymbionts, fungal endosymbiont has been identified using PCR method in planthopper, Ricania japonica [15]. This yeast-like endosymbiont uses the enzyme uricase to recycle uric acid secreted by the host spe- cies, assisting in metabolic processes [15]. In addition, yeast-like symbionts have been identified in Nilaparvata lugens, a brown planthopper $[16,17]$ which also support the host's uric acid metabolism [18]. However, there was no sequence information of this endosymbiont until the complete fungal mitogenome was obtained from the raw reads 


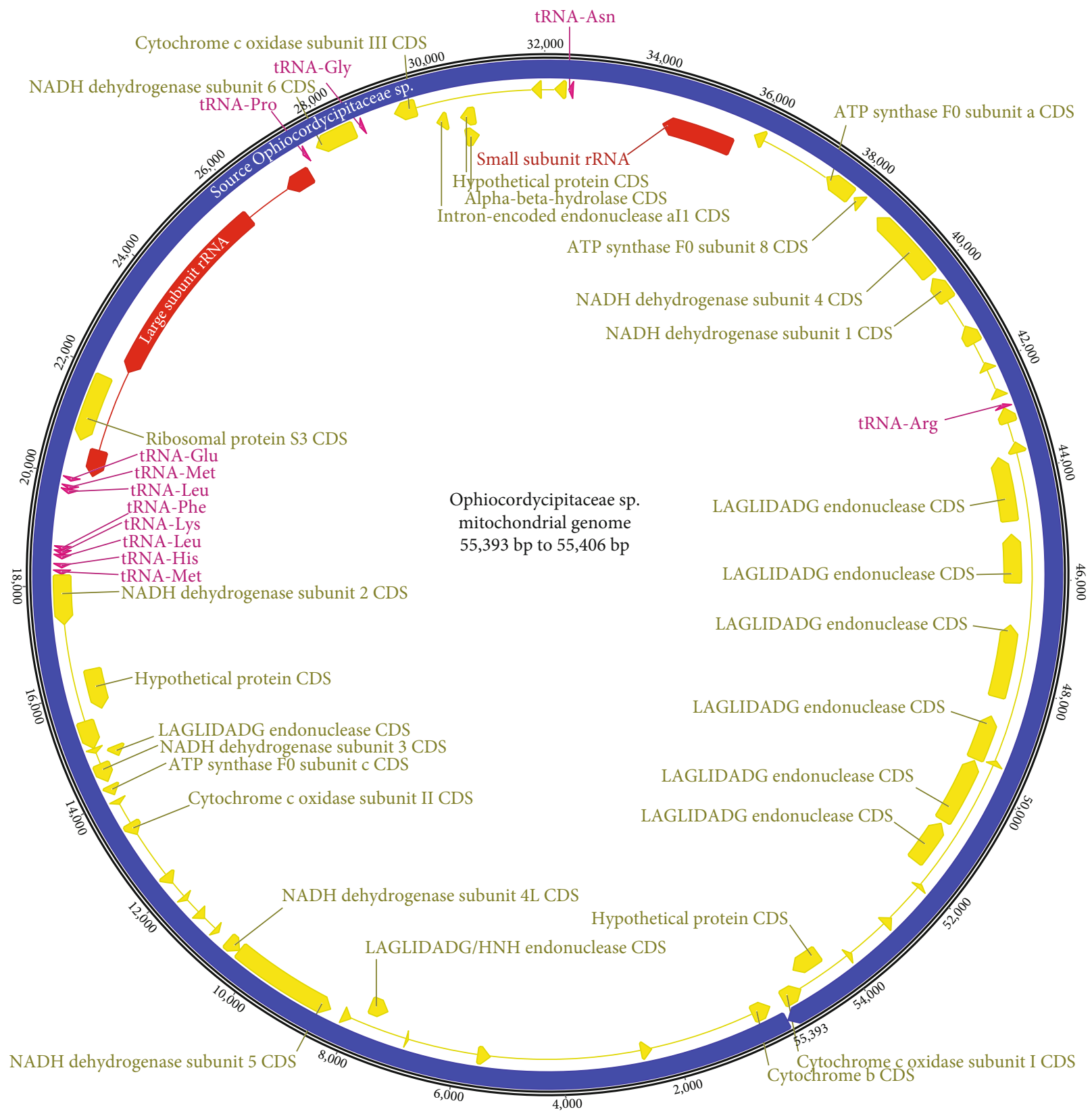

FIGURE 1: Complete mitogenome of WBPH fungal endosymbionts. Blue circle indicates fungal endosymbiont mitogenome, yellow arrows are protein-coding genes, purple arrows are tRNAs, and red arrows mean rRNAs. Direction of arrows indicates direction of transcription. Each gene name was displayed with lines directing to the corresponded arrows. Numbers displayed outside of blue circle mean base pair.

of Ricania speculum, a black planthopper [19]. This mitogenome was identified as an Ophiocordycipitaceae species by comparing already known several complete mitogenomes in this family [19]. This result suggests that next-generation sequencing technology that provides a large number of short reads can be used to provide evidence for the existence of endosymbiont species using DNA extracted from insect species. These results draw comparison to previous studies that have successfully identified a multiple number of complete organelle or bacterial genomes from one NGS library [12, 19-37].

Here, we reported the first complete mitogenomes of fungal WBPH endosymbiont from five WBPH samples isolated in Korea and China. The five mitogenomes display 55,390 to 55,406 bp in length, shorter than that of $R$. speculum [19]. The numbers of intraspecific variations among the five mitogenomes are fewer in number than those of the four Ophiocordycipitaceae species. Phylogenetic analysis based on conserved PCGs across Ophiocordycipitaceae mitogenomes displays that the five mitogenomes were clustered with that of $R$. speculum, forming an independent clade. Once additional planthopper fungal endosymbiont mitogenomes become available, their phylogenetic relationships as well as evolutionary histories based on their complete mitogenomes will become clearer. 


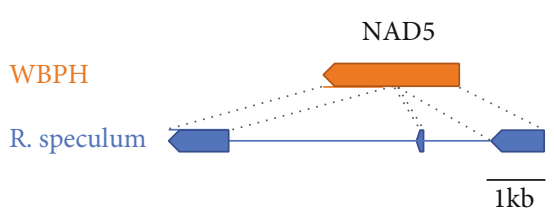

(a)

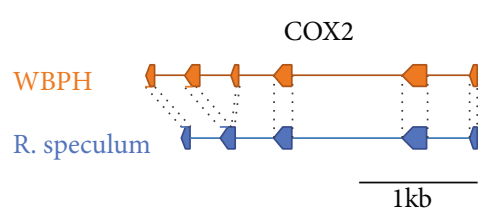

(b)

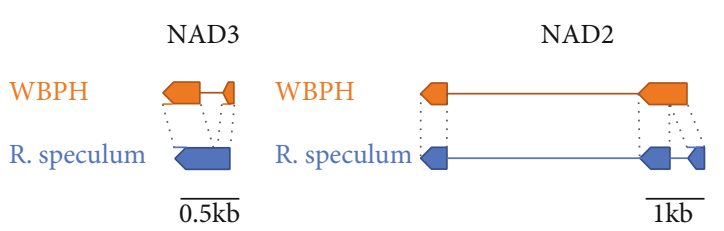

(c)

(d)

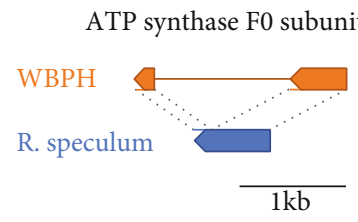

(e)
COX1

\section{WBPH \\ R. speculum}

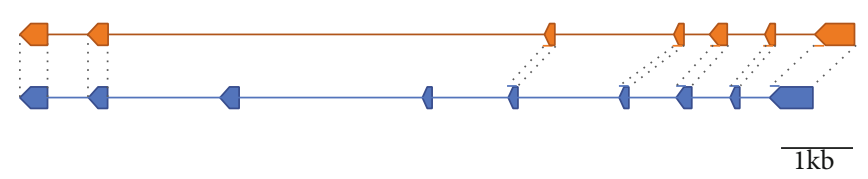

(f)

FIgURE 2: Schematic diagram of exon/intron structure of protein-coding genes displaying different configurations between fungal endosymbiont mitogenomes of WBPH and R. speculum. Orange-colored diagrams indicate components of WBPH fungal endosymbionts, and blue-colored diagrams are those of R. speculum fungal endosymbionts. Exon/intron structures of (a) NAD5, (b) COX2, (c) NAD3, (d) NAD2, (e) ATP synthase F0 subunit, and (f) COX1 are displayed.

TABLE 3: List of intraspecific variations identified on the five WBPH fungal endosymbiont mitogenomes.

\begin{tabular}{lccccc}
\hline No. & Type & Coordination of multiple sequence alignments & Strains & Base changes & Position \\
\hline 1 & Insertion & $4209-4210$ & KR.1D, KR.5D & - to CC & Intergenic \\
2 & Insertion & $27,476-27,486$ & KR.1D & - to TGGGCCCCCC & Intergenic \\
3 & SNP & 27,487 & KR.1D & A to C & Intergenic \\
4 & Deletion & $32,727-32,728$ & KR.5D & CC to - & Intergenic \\
5 & SNP & 37,574 & KR.5D & A to T & L to Q in ATP synthase F0 subunit \\
6 & Insertion & 38,727 & KR.1D & - to G & Intergenic \\
7 & Deletion & $38,728-38,730$ & KR.5D & GGG to - & Intergenic \\
\hline
\end{tabular}

\section{Materials and Methods}

2.1. DNA Preparation and Genome Sequencing of Four WBPH Samples. All four WBPH samples were captured at two places in Korea (Table 1). One individual of WBPH was frozen with liquid nitrogen using $1.5 \mathrm{ml}$ microtube and then was ground using a plastic pestle. The Quick-DNA Miniprep Plus Kit (Zymo Research, USA) was used for extracting DNA. Genome sequencing was performed using NovaSeq6000 at Macrogen Inc., Korea, from the extracted DNA of four WBPH samples with constructing a $350 \mathrm{bp}$ pair-end library.

2.2. Assembly and Annotation of the Five Fungal WBPH Endosymbiont Mitogenomes. De novo assembly, with confirmation, was accomplished with Velvet v1.2.10 [38] after filtering raw reads using Trimmomatic v0.33 [39]. After obtaining mitogenome contig sequences with the condition that sequence coverage is more than $60 \mathrm{x}$, gaps were filled with GapCloser v1.12 [40], and all bases from the assembled sequences were confirmed by checking each base in the align- ment (tview mode in SAMtools v1.9 [41]) against the assembled mitogenome generated with BWA v0.7.17 [42]. The circular form of mitogenomes was confirmed by the pairend reads connecting both sides of mitogenomes. All these bioinformatic analyses were conducted under the environment of the Genome Information System (GeIS; http://geis .infoboss.co.kr/) like the previous studies of mitogenomes $[19,21-24,26,28,30,32,33,36,43-91]$.

Geneious Prime ${ }^{\circledR}$ 2020.2.4 (Biomatters Ltd, Auckland, New Zealand) was used for mitogenome annotation with referring to the mitogenome of $R$. speculum fungal endosymbiont (NC_ 049089) [19] by transferring annotations while correcting exceptional cases, including missing start or stop codons. Also, the "FindORF" function in Geneious Prime ${ }^{\circledR} 2020.2 .4$ together with BLAST v2.2.24 [92] was also utilized to find novel PCGs including LAGLIDADG endonucleases. tRNAs were predicted and confirmed using tRNAScan-SE v2.0.6 [93].

2.3. Identification of Sequence Variations on the Complete Mitogenomes of WBPH Fungal Endosymbionts. Single- 
TABLE 4: List of available complete fungal mitogenomes in Ophiocordycipitaceae.

\begin{tabular}{|c|c|c|c|c|c|c|c|c|}
\hline No. & Species & NCBI accession & Length (bp) & GC ratio (\%) & No. of PCGs & No. of tRNAs & No. of rRNAs & Reference \\
\hline 1 & Ophiocordyceps sinensis & NC_034659 & 157,539 & 30.2 & 88 & 27 & 2 & Unpub. \\
\hline 2 & Ophiocordyceps sinensis & MH400233 & 157,584 & 30.2 & 76 & 27 & 2 & Unpub. \\
\hline 3 & Ophiocordyceps sinensis & KP835313 & 157,510 & 30.2 & $6^{*}$ & 23 & 2 & {$[123]$} \\
\hline 4 & Hirsutella thompsonii & NC_040165 & 62,509 & 29.8 & 30 & 27 & 2 & [114] \\
\hline 5 & Hirsutella thompsonii & MH367296 & 65,332 & 30.3 & 32 & 27 & 2 & [114] \\
\hline 6 & Hirsutella thompsonii & MH367295 & 60,362 & 30.0 & 29 & 27 & 2 & [114] \\
\hline 7 & Hirsutella rhossiliensis & MG979071 & 62,949 & 28.3 & 33 & 26 & 2 & [129] \\
\hline 8 & Hirsutella rhossiliensis & NC_030164 & 62,483 & 28.2 & 24 & 26 & 2 & Unpub. \\
\hline 9 & Hirsutella vermicola & NC_036610 & 53,793 & 25.3 & 27 & 25 & 2 & {$[130]$} \\
\hline 10 & Hirsutella minnesotensis & NC_027660 & 52,245 & 28.4 & 30 & 25 & 2 & [131] \\
\hline 11 & Tolypocladium sp. & MN583265 & 46,466 & 26.1 & 15 & 26 & 2 & [132] \\
\hline 12 & Tolypocladium guangdongense & MT471267 & 46,102 & 26.1 & 30 & 27 & 2 & [133] \\
\hline 13 & Tolypocladium ophioglossoides & NC_031384 & 35,159 & 27.5 & 19 & 25 & 2 & {$[134]$} \\
\hline 14 & Tolypocladium cylindrosporum & NC_046839 & 34,698 & 27,0 & 24 & 26 & 2 & [135] \\
\hline 15 & Tolypocladium inflatum & NC_036382 & 25,328 & 27.8 & 15 & 25 & 2 & {$[136]$} \\
\hline 16 & Tolypocladium inflatum & KY924880 & 25,238 & 27.8 & 15 & 25 & 2 & {$[136]$} \\
\hline 17 & Tolypocladium inflatum & KY924881 & 25,328 & 27.8 & 15 & 25 & 2 & {$[136]$} \\
\hline 18 & Tolypocladium inflatum & KY924882 & 25,328 & 27.8 & 15 & 25 & 2 & {$[136]$} \\
\hline 19 & Tolypocladium inflatum & KY924883 & 24,793 & 27.8 & 15 & 25 & 2 & {$[136]$} \\
\hline 20 & Ophiocordycipitaceae sp. & NC_049089 & 66,785 & 30.6 & 31 & 17 & 2 & [19] \\
\hline
\end{tabular}

${ }^{*}$ Mitogenome annotation of this genome (KP835313) seems not to be complete because several major genes, such as COX1, NAD1, NAD5, and COB, which have many introns on fungal mitogenomes that were not annotated as CDS.

TABLE 5: Number of intraspecific variations identified from four fungal species belonging to Ophiocordycipitaceae.

\begin{tabular}{|c|c|c|c|c|c|c|c|}
\hline No. & Species & $\begin{array}{c}\text { No. of } \\
\text { mitogenomes }\end{array}$ & $\begin{array}{l}\text { Aligned length } \\
\text { (bp) }\end{array}$ & $\begin{array}{l}\text { No. of } \\
\text { SNPs }\end{array}$ & $\begin{array}{c}\text { SNP coverage } \\
(\%)\end{array}$ & $\begin{array}{l}\text { No. of } \\
\text { INDELs }\end{array}$ & $\begin{array}{c}\text { INDEL coverage } \\
(\%)\end{array}$ \\
\hline 1 & $\begin{array}{l}\text { Ophiocordyceps } \\
\text { sinensis }\end{array}$ & 3 & 157,606 & 16 & 0.010 & 144 & 0.091 \\
\hline 2 & Hirsutella thompsonii & 3 & 66,635 & 281 & 0.42 & 6489 & 9.74 \\
\hline 3 & Hirsutella rhossiliensis & 2 & 64,858 & 7 & 0.01 & 2008 & 3.10 \\
\hline 4 & $\begin{array}{l}\text { Tolypocladium } \\
\text { inflatum }\end{array}$ & 5 & 25,338 & 30 & 0.12 & 375 & 1.48 \\
\hline
\end{tabular}

nucleotide polymorphisms (SNPs) and insertions and deletions (INDELs) were identified using the "Find variations/SNP" function implemented in Geneious Prime ${ }^{\circledR}$ 2020.2.4 (Biomatters Ltd, Auckland, New Zealand) based on multiple sequence alignment of the five mitogenomes of WBPH fungal endosymbionts conducted by MAFFT v7.450 [94]. Each identified variation was manually checked to understand which mitogenome has them.

2.4. Identification of Simple Sequence Repeats (SSRs). Simple sequence repeats (SSRs) were identified on the mitogenome sequence using the pipeline of the SSR database (SSRDB; http://ssr.pe.kr/; Park et al., in preparation). Based on the conventional definition of an SSR on an organelle genome, monoSSR ( $1 \mathrm{bp}$ ) to hexaSSR (6 bp), the total length of SSRs on the mitogenome exceeds $10 \mathrm{bp}$. Owing to the different criteria of SSRs on organelle genomes [95-101], we adopted the criteria used in various organelle genome analyses [21, 44, 102-104], as follows: the monoSSR (unit sequence length of $1 \mathrm{bp}$ ) to hexaSSR (6 bp) are used as normal SSRs, and heptaSSR ( $7 \mathrm{bp})$ to decaSSR ( $10 \mathrm{bp}$ ) are defined as extended SSRs. Among the normal SSRs, pentaSSRs and hexaSSRs for which the number of unit sequences is 2 are classified as potential SSRs.

2.5. Construction of Phylogenetic Trees. Five conserved PCGs, including $A T P 8, C O 2, N A D 3, N A D 4$, and $N A D 4 L$, from 26 fungal mitogenomes including the five mitogenomes assembled in this study and one outgroup species, Fusarium graminearum, were aligned independently using MAFFT v7.450 [94] and concatenated using the Perl script, one of the component of GenomeArchive ${ }^{\circledR}$ (http://www.genomearchive .info) [105]. The model test was conducted with jModelTest v2.1.5 [106]. The neighbor-joining (NJ) and maximum- 


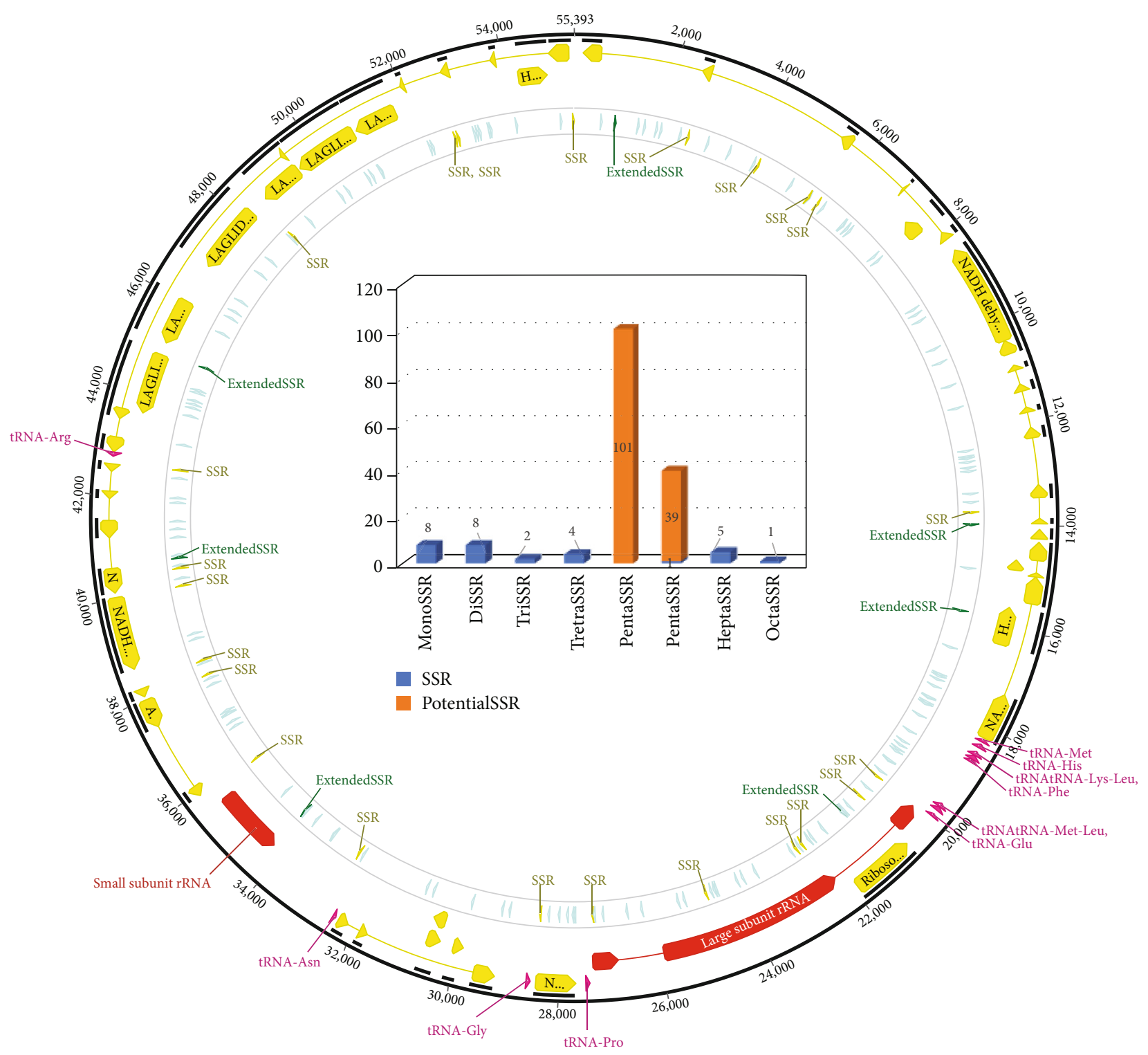

FIGURE 3: Numbers and distribution of SSRs on the mitogenome of fungal endosymbiont of WBPH KR. (a) Black circle indicates fungal endosymbiont mitogenome, yellow arrows are protein-coding genes, purple arrows are tRNAs, and red arrows mean rRNAs. SSRs, extended SSRs, and potential SSRs are displayed with yellow, green, and light green colors, respectively. (b) The number of SSRs along with SSR types is displayed. Blue color indicates SSRs and extended SSRs, and orange color means potential SSRs.

likelihood (ML) trees were reconstructed in MEGA X [107]. In the ML analysis, a heuristic search was used with nearest-neighbor interchange (NNI) branch swapping, general time reversible (GTR) model, and uniform rates among sites. All other options used the default settings. Bootstrap analyses with 1000 pseudoreplicates were conducted with the same options. The posterior probability of each node was estimated by Bayesian inference (BI) using the MrBayes v3.2.7 [108] plug-in implemented in Geneious Prime ${ }^{\circledR}$ 2020.2.4. The HKY85 model with gamma rates was used as a molecular model. A Markov chain Monte Carlo (MCMC) algorithm was employed for 1,100,000 generations, sampling trees every 200 generations, with four chains running simultaneously. Trees from the first 100,000 generations were discarded as burn-in.

\section{Results and Discussions}

3.1. Complete Mitogenome of Fungal WBPH Endosymbionts. We successfully assembled fungal endosymbiont mitogenomes from four WBPH samples isolated in Korea and China and one public dataset of NGS raw reads (Table 1). This is the first WBPH fungal endosymbiont mitogenome identified. Their lengths ranged from $55,390 \mathrm{bp}$ to $55,406 \mathrm{bp}$ (Table 1), which is shorter than that of R. speculum (66,785 bp) [19]. In these mitogenomes, there were 28 protein-coding genes (PCGs), 12 tRNAs, and 2 rRNAs (Table 2). Some of the PCGs found were LAGLIDADG endonucleases, which are usually found in intronic regions of various fungal mitogenomes, contributing to the expansion of their length $[19,55,108-$ 112]. In comparison to the previously sequenced 
TABLE 6: List of SSRs identified from the five fungal mitogenomes of WBPH endosymbionts.

\begin{tabular}{lccccc}
\hline SSR type & KR & KR.1D & KR.5D & KR.11D & WGS \\
\hline MonoSSR & 8 & 9 & 8 & 8 & 8 \\
DiSSR & 8 & 8 & 8 & 8 & 8 \\
TriSSR & 2 & 2 & 2 & 2 & 2 \\
TetraSSR & 4 & 4 & 4 & 4 & 4 \\
PentaSSR & 0 & 0 & 0 & 0 & 0 \\
HexaSSR & 1 & 1 & 1 & 1 & 1 \\
HeptaSSR & 5 & 5 & 5 & 5 & 5 \\
OctaSSR & 1 & 1 & 1 & 1 & 1 \\
NonaSSR & 0 & 0 & 0 & 0 & 0 \\
DecaSSR & 0 & 0 & 0 & 0 & 0 \\
Subtotal & 29 & 30 & 29 & 29 & 29 \\
PentaPotentialSSR & 101 & 101 & 101 & 101 & 101 \\
HexaPotentialSSR & 39 & 39 & 39 & 39 & 39 \\
Subtotal & 140 & 140 & 140 & 140 & 140 \\
\hline
\end{tabular}

mitogenome of the fungal endosymbiont of $R$. speculum, there were slightly fewer PCGs and tRNAs found in the WBPH endosymbiont mitogenomes. There were three fewer PCGs for three reasons: the smaller number of LAGLIDADG endonucleases, the absence of one endonuclease and a GIYYIG endonuclease, and the presence of two additional PCGs-a hypothetical protein and a LAGLIDADG/HNH endonuclease. This particular configuration of PCGs is usually identified in other fungal mitogenomes; for example, two mitogenomes of Fusarium oxysporum (GenBank accessions are MN259514 and MN259515) display two completely different PCGs in each mitogenome $[54,56]$. There are also five fewer tRNAs because of the different configurations: tRNA-Asp, tRNA-Cys, tRNA-Ile, and two tRNA-Ser (also found in the mitogenome of the fungal symbiont of R. speculum [19]). This difference in configuration of tRNAs between two different fungal symbionts suggests that tRNA configuration may not be critical because essential tRNAs absent in the fungal mitogenome can be supported from the nuclear genome [113].

Several PCGs in the fungal mitogenomes have been invaded by introns multiple times. For example, COX1 contains three introns, and $C O B$ has five introns in the Hirsutella thompsonii mitogenome [114]. This phenomenon contributes to increased fungal mitogenome: Aspergillus pseudoglaucus and Aspergillus egyptiacus are longer than the other Aspergillus mitogenomes because of the presence of many introns on major PCGs $[55,115]$. The fungal mitogenomes examined in this study also present many introns on PCGs including $C O B, C O X 1, N A D 1, A T P 8, C O X 3, C O X 2$, and NAD2 (Figure 1), which is a major reason for the expansion of fungal mitogenomes together with endonucleases.

The gene order of WBPH and R. speculum fungal symbiont mitogenomes was the same when PCGs except endonucleases and rRNAs are considered. However, intron structures of COX1, COX2, NAD2, NAD3, NAD5, and ATP synthase F0 subunit present different configurations between the two mitogenomes (Figure 2). The intron structures of NAD5 and NAD2 present reduce of a reduction in the number of exons via removal of intron regions in the WBPH fungal endosymbiont mitogenome (Figures 2(a) and 2(d)), whereas those of COX2, NAD3, and the ATP synthase F0 subunit display insertions of one intron into the WBPH fungal endosymbiont mitogenome (Figures 2(b), 2(c), and 2(e)). This indicates that the reduction in the total length of the WBPH fungal symbiont mitogenome is not primarily caused by reducing the number of exons, unlike in Aspergillus mitogenomes $[55,116]$. In addition, COX1, which contains the largest number of exons in these mitogenomes, lost the sixth and seventh exons of the $R$. speculum fungal endosymbiont mitogenome in the mitogenome of WBPH endosymbiont (Figure 2(f)). However, the total length of COX1, including the introns of WBPH fungal endosymbionts, is longer than that of $R$. speculum fungal endosymbionts by $1 \mathrm{~kb}$ (Figure 2(f)), reflecting complex events that occurred during the evolution of both mitogenomes. Additional studies are required to identify the correct exons of the COX1 gene of this fungal endosymbiont. For example, alignment of RNASeq raw reads against this mitogenome could provide expressed regions in this mitogenome.

Once more fungal symbiont mitogenomes are available, patterns of presence and absence of tRNAs, additional endonucleases, and intron structures of PCGs in endosymbiont mitogenomes will elucidate a detailed evolutionary history of these genes.

\subsection{Identification of Intraspecific Variations on Fungal} WBPH Endosymbiont Mitogenomes. We identified two SNPs, three insertions, and two deletions via multiple sequence alignments of the five fungal mitogenomes (Table 3). One of two SNPs was identified in KR.5D WBPH and changed leucine (L) to glutamine $(\mathrm{Q})$ in the ATP synthase F0 subunit (Table 3). One $10 \mathrm{bp}$ insertion in the intergenic space was found in KR.1D WBPH, while the remaining two insertions and all three deletions were 1 to $3 \mathrm{bp}$ in length (Table 3 ).

The proportions of these intraspecific SNPs, insertions, and deletions in these fungal mitogenomes were $0.0036 \%$, $0.020 \%$, and $0.012 \%$, respectively. The proportion of insertions and deletions was higher than that of SNPs. Interestingly, there is geographical variation in the fungal symbiont mitogenomes. The mitogenome of WBPH endosymbionts used in the whole genome sequencing (WGS) and the KR.11D isolate were identical to that of KR, while the other three WBPHs captured in other locations in Korea displayed intraspecific variations. The sample used in the WGS originated from the University of Science and Technology of China (Anhui province, China), indicating that KR 11D and KR WBPH samples obtained in Korea have migrated from the similar region to the WGS sample. However, further analyses of their complete mitogenomes or whole genomes will be needed to provide more supportive data for identifying their origins.

There is a relatively small number of intraspecific SNPs and INDELs identified from these fungal mitogenomes in comparison to those of other fungal mitogenomes, for 


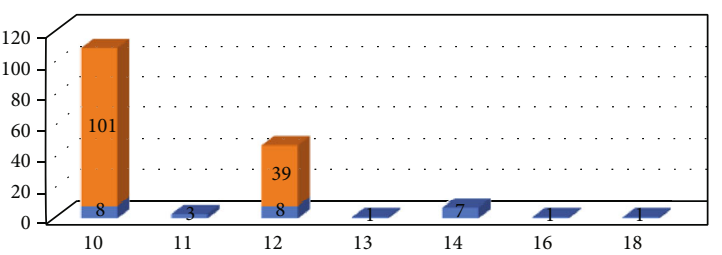

(a)

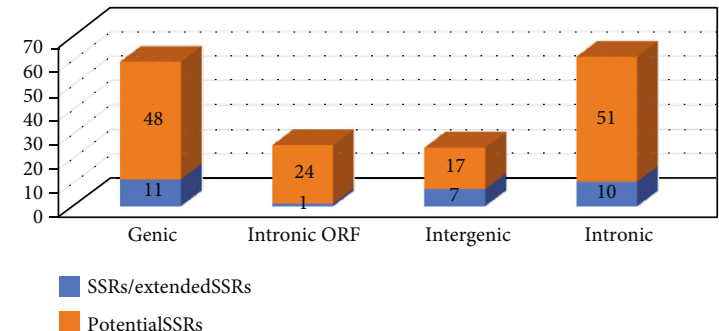

(b)

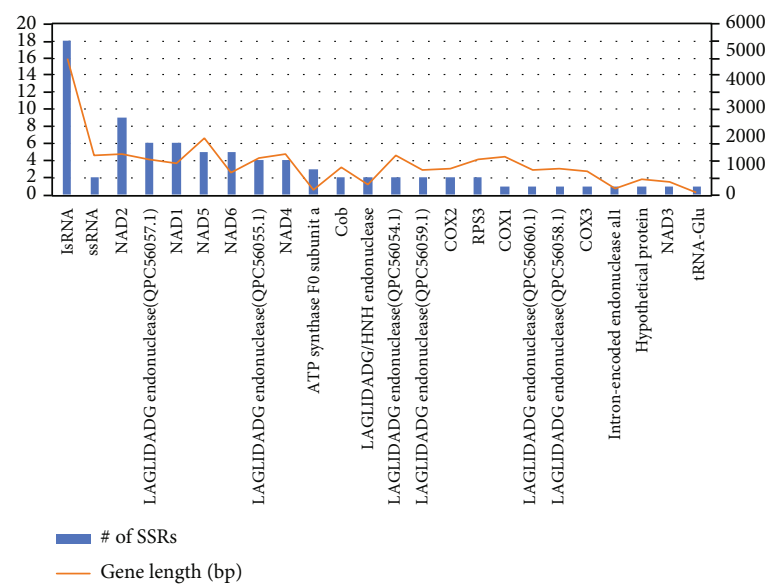

(c)

FIGURE 4: Comparative analysis of simple sequence repeats on mitogenome of WBPH fungal endosymbiont. (a) Number of SSRs, extended SSRs, and potential SSRs along with its length. $x$-axis indicates SSR length (bp), and $y$-axis means the number of SSRs. Blue-colored bars indicate SSRs and extended SSRs, and orange bars mean potential SSRs. (b) Number of SSRs, extended SSRs, and potential SSRs based on positions, genic, intronic ORF, intergenic, and intronic. (c) Number of genic SSRs along with genes (blue bars) as well as length of genes (orange line). $x$-axis is genes containing SSRs, and $y$-axis indicates the number of SSRs (left) and gene length (bp; right).

example, 16 to 17 SNPs ( $0.055 \%$ to $0.0582 \%$ ) and 22 to 27 INDELs $(0.075 \%$ to $0.092 \%)$ on Aspergillus flavus [52, 53] and 62 SNPs $(0.15 \%)$ and 181 INDELs (0.43\%) on Fusarium oxysporum f.sp. lactucae [56]. They are also fewer than those identified in insect mitogenomes [10, 22, 23, 43, 45-51].

Based on 25 available complete fungal mitogenomes in Ophiocordycipitaceae, four species, Ophiocordyceps sinensis, Hirsutella thompsonii, Hirsutella rhossiliensis, and Tolypocladium inflatum, contain more than one complete fungal mitogenome (Table 4). We investigated intraspecific variations in the mitogenomes of these four species (Table 5). There are significantly more INDELs than SNPs identified in the four fungal species, a trend identical to that observed in the four mitogenomes of fungal endosymbiont WBPH with the exception of their absolute amounts. Moreover, there were at least three times more SNPs and INDELs in these fungal mitogenomes than that in the fungal symbiont of WBPHs. This phenomenon can be explained by two major factors: first, the geographical distribution or genetic background of WBPH samples is relatively limited in comparison to those of the four fungal species, and second, the surroundings of fungal endosymbionts are less dynamic than those of normal fungal species, causing low selection pressure from the environment. This second factor is supported by two studies: first, the bacterial genome of aphid endosymbiont Buchnera aphidicola (Aphis gossypii) displays a low level of intraspecific variation in comparison to those of host mitogenome (Bae et al., under revision), and second, the whole genome of endosymbiont of Pediculus humanus capitis also shows low-level intraspecific variations in comparison to those of their whole genomes [117].

3.3. Identification and Comparative Analysis of Simple Sequence Repeats on the Five WBPH Fungal Endosymbiont Mitogenomes. Simple sequence repeats (SSRs) identified from organellar genomes have been utilized as molecular markers in various species such as plant species [99, 118122], suggesting that SSRs on fungal endosymbiont mitogenomes can be used as molecular markers to identify the geographical origins of WBPH. In total, 23 normal and 6 extended SSRs were identified from fungal endosymbiont mitogenomes (Figure 3(b)), with the exception of the fungal endosymbiont mitogenome of WBPH KR.1D which displays 24 normal and 6 extended SSRs (Table 6). The fungal endosymbiont mitogenome of WBPH KR.1D has one more monoSSR (Table 6) with a unit sequence of $\mathrm{C}$ and length of $15 \mathrm{bp}$ caused by one insertion (Table 3). In addition, 140 potential SSRs were also identified in the five mitogenomes (Table 6). SSRs identified in the mitogenome were distributed evenly (Figure 3(a)), suggesting that there was no hot spot of SSRs in these fungal mitogenomes. 
TABLE 7: List of SSRs identified on fungal endosymbiont mitogenome of WBPH KR.

\begin{tabular}{|c|c|c|c|c|c|c|c|c|}
\hline No. & Name & SSR type & Type & Start & End & $\begin{array}{c}\text { Unit } \\
\text { sequence }\end{array}$ & $\begin{array}{l}\text { Repeat } \\
\text { number }\end{array}$ & Genes \\
\hline 1 & M0000001 & Normal SSR & MonoSSR & 4209 & 4219 & $\mathrm{C}$ & 11 & (Intron)Cob \\
\hline 2 & M0000002 & Normal SSR & MonoSSR & 5548 & 5558 & A & 11 & Cob \\
\hline 3 & M0000003 & Normal SSR & MonoSSR & 22314 & 22323 & $\mathrm{~T}$ & 10 & Large subunit ribosomal RNA \\
\hline 4 & M0000004 & Normal SSR & MonoSSR & 22482 & 22492 & $\mathrm{~T}$ & 11 & Large subunit ribosomal RNA \\
\hline 5 & M0000005 & Normal SSR & MonoSSR & 28442 & 28451 & $\mathrm{~T}$ & 10 & \\
\hline 6 & M0000006 & Normal SSR & MonoSSR & 32715 & 32728 & $\mathrm{C}$ & 14 & \\
\hline 7 & M0000007 & Normal SSR & MonoSSR & 38315 & 38327 & G & 13 & \\
\hline 8 & M0000008 & Normal SSR & MonoSSR & 40035 & 40044 & $\mathrm{~T}$ & 10 & NAD4 \\
\hline 9 & D0000001 & Normal SSR & DiSSR & 5788 & 5797 & TA & 5 & (Intron)Cob \\
\hline 10 & D0000002 & Normal SSR & DiSSR & 13708 & 13721 & AT & 7 & (Intron) COX2 \\
\hline 11 & D0000003 & Normal SSR & DiSSR & 20068 & 20077 & AT & 5 & Large subunit ribosomal RNA \\
\hline 12 & D0000004 & Normal SSR & DiSSR & 20646 & 20657 & AT & 6 & (Intron)large subunit ribosomal RNA \\
\hline 13 & D0000005 & Normal SSR & DiSSR & 27266 & 27275 & TA & 5 & Large subunit ribosomal RNA \\
\hline 14 & D0000006 & Normal SSR & DiSSR & 37995 & 38004 & TA & 5 & ATP synthase F0 subunit a \\
\hline 15 & D0000007 & Normal SSR & DiSSR & 40430 & 40441 & $\mathrm{AT}$ & 6 & NAD1 \\
\hline 16 & D0000008 & Normal SSR & DiSSR & 55352 & 55361 & TA & 5 & \\
\hline 17 & T0000001 & Normal SSR & TriSSR & 35829 & 35840 & ATT & 4 & \\
\hline 18 & T0000002 & Normal SSR & TriSSR & 52780 & 52791 & ATA & 4 & (Intron)COX1 \\
\hline 19 & $\mathrm{Te} 0000001$ & Normal SSR & TetraSSR & 24721 & 24732 & ATTT & 3 & Large subunit ribosomal RNA \\
\hline 20 & $\mathrm{Te} 0000002$ & Normal SSR & TetraSSR & 42598 & 42609 & TTTA & 3 & \\
\hline 21 & $\mathrm{Te} 0000003$ & Normal SSR & TetraSSR & 48477 & 48488 & ATAA & 3 & (Intron)COX1 \\
\hline 22 & Te0000004 & Normal SSR & TetraSSR & 52709 & 52720 & AATA & 3 & (Intron)COX1 \\
\hline 23 & P0000001 & Potential SSR & PentaSSR & 586 & 595 & TTGT & 2 & (Intron)Cob \\
\hline 24 & P0000002 & Potential SSR & PentaSSR & 1923 & 1932 & TAATA & 2 & (Intron)Cob \\
\hline 25 & P0000003 & Potential SSR & PentaSSR & 3523 & 3532 & TAAAA & 2 & (Intron)Cob \\
\hline 26 & P0000004 & Potential SSR & PentaSSR & 4116 & 4125 & TTGTC & 2 & (Intron)Cob \\
\hline 27 & P0000005 & Potential SSR & PentaSSR & 5068 & 5077 & ATAAT & 2 & (Intron)Cob \\
\hline 28 & P0000006 & Potential SSR & PentaSSR & 6540 & 6549 & TAATG & 2 & (Intron)Cob \\
\hline 29 & P0000007 & Potential SSR & PentaSSR & 6646 & 6655 & ATTTT & 2 & (Intron)Cob \\
\hline 30 & P0000008 & Potential SSR & PentaSSR & 6714 & 6723 & TTTT & 2 & (Intron)Cob \\
\hline 31 & P0000009 & Potential SSR & PentaSSR & 7636 & 7645 & AGCAA & 2 & $\begin{array}{c}\text { LAGLIDADG/HNH } \\
\text { endonuclease, (Intron)Cob }\end{array}$ \\
\hline 32 & P0000010 & Potential SSR & PentaSSR & 9109 & 9118 & AAGTT & 2 & NAD5 \\
\hline 33 & P0000011 & Potential SSR & PentaSSR & 9264 & 9273 & ATAA & 2 & NAD5 \\
\hline 34 & P0000012 & Potential SSR & PentaSSR & 10305 & 10314 & AGACA & 2 & NAD5 \\
\hline 35 & P0000013 & Potential SSR & PentaSSR & 10857 & 10866 & ATTCA & 2 & \\
\hline 36 & P0000014 & Potential SSR & PentaSSR & 11369 & 11378 & AGATA & 2 & $\mathrm{COX} 2$ \\
\hline 37 & P0000015 & Potential SSR & PentaSSR & 12511 & 12520 & TTATA & 2 & (Intron)COX2 \\
\hline 38 & P0000016 & Potential SSR & PentaSSR & 12600 & 12609 & TAAGA & 2 & (Intron)COX2 \\
\hline 39 & P0000017 & Potential SSR & PentaSSR & 12710 & 12719 & AAGCG & 2 & (Intron)COX2 \\
\hline 40 & P0000018 & Potential SSR & PentaSSR & 12796 & 12805 & TTAAC & 2 & (Intron)COX2 \\
\hline 41 & P0000019 & Potential SSR & PentaSSR & 13303 & 13312 & TAATA & 2 & $\mathrm{COX} 2$ \\
\hline 42 & P0000020 & Potential SSR & PentaSSR & 14946 & 14955 & AAAAG & 2 & NAD3 \\
\hline 43 & P0000021 & Potential SSR & PentaSSR & 16747 & 16756 & TCGAG & 2 & (Intron)NAD2 \\
\hline 44 & P0000022 & Potential SSR & PentaSSR & 17383 & 17392 & TCATT & 2 & NAD2 \\
\hline 45 & P0000023 & Potential SSR & PentaSSR & 17427 & 17436 & AATAA & 2 & NAD2 \\
\hline 46 & P0000024 & Potential SSR & PentaSSR & 17514 & 17523 & AAATG & 2 & NAD2 \\
\hline 47 & P0000025 & Potential SSR & PentaSSR & 17685 & 17694 & AATAA & 2 & NAD2 \\
\hline
\end{tabular}


TABle 7: Continued.

\begin{tabular}{|c|c|c|c|c|c|c|c|c|}
\hline No. & Name & SSR type & Type & Start & End & $\begin{array}{c}\text { Unit } \\
\text { sequence }\end{array}$ & $\begin{array}{l}\text { Repeat } \\
\text { number }\end{array}$ & Genes \\
\hline 48 & P0000026 & Potential SSR & PentaSSR & 18083 & 18092 & AATAC & 2 & NAD2 \\
\hline 49 & P0000027 & Potential SSR & PentaSSR & 18109 & 18118 & TATT & 2 & NAD2 \\
\hline 50 & P0000028 & Potential SSR & PentaSSR & 18121 & 18130 & ATAGA & 2 & NAD2 \\
\hline 51 & P0000029 & Potential SSR & PentaSSR & 18400 & 18409 & TTATG & 2 & \\
\hline 52 & P0000030 & Potential SSR & PentaSSR & 18819 & 18828 & GATA & 2 & \\
\hline 53 & P0000031 & Potential SSR & PentaSSR & 19078 & 19087 & ATTTT & 2 & \\
\hline 54 & P0000032 & Potential SSR & PentaSSR & 19190 & 19199 & TTGTA & 2 & \\
\hline 55 & P0000033 & Potential SSR & PentaSSR & 19302 & 19311 & ATAAT & 2 & \\
\hline 56 & P0000034 & Potential SSR & PentaSSR & 19613 & 19622 & AACT & 2 & \\
\hline 57 & P0000035 & Potential SSR & PentaSSR & 19629 & 19638 & TATT & 2 & \\
\hline 58 & P0000036 & Potential SSR & PentaSSR & 19904 & 19913 & TAGAC & 2 & tRNA-Glu \\
\hline 59 & P0000037 & Potential SSR & PentaSSR & 20384 & 20393 & TTATT & 2 & Large subunit ribosomal RNA \\
\hline 60 & P0000038 & Potential SSR & PentaSSR & 20968 & 20977 & TTATT & 2 & RPS3, (Intron)large subunit ribosomal RNA \\
\hline 61 & P0000039 & Potential SSR & PentaSSR & 21146 & 21155 & TGTAT & 2 & RPS3, (Intron)large subunit ribosomal RNA \\
\hline 62 & P0000040 & Potential SSR & PentaSSR & 21173 & 21182 & TATTA & 2 & RPS3, (Intron)large subunit ribosomal RNA \\
\hline 63 & P0000041 & Potential SSR & PentaSSR & 21730 & 21739 & TTATT & 2 & RPS3, (Intron)large subunit ribosomal RNA \\
\hline 64 & P0000042 & Potential SSR & PentaSSR & 22038 & 22047 & TTTTA & 2 & (Intron)large subunit ribosomal RNA \\
\hline 65 & P0000043 & Potential SSR & PentaSSR & 22121 & 22130 & TTATT & 2 & (Intron)large subunit ribosomal RNA \\
\hline 66 & P0000044 & Potential SSR & PentaSSR & 22606 & 22615 & TAATA & 2 & Large subunit ribosomal RNA \\
\hline 67 & P0000045 & Potential SSR & PentaSSR & 23512 & 23521 & AAGAC & 2 & Large subunit ribosomal RNA \\
\hline 68 & P0000046 & Potential SSR & PentaSSR & 23962 & 23971 & TTTTC & 2 & Large subunit ribosomal RNA \\
\hline 69 & P0000047 & Potential SSR & PentaSSR & 24453 & 24462 & AATTA & 2 & Large subunit ribosomal RNA \\
\hline 70 & P0000048 & Potential SSR & PentaSSR & 24501 & 24510 & ATTTA & 2 & Large subunit ribosomal RNA \\
\hline 71 & P0000049 & Potential SSR & PentaSSR & 25211 & 25220 & TTTAC & 2 & Large subunit ribosomal RNA \\
\hline 72 & P0000050 & Potential SSR & PentaSSR & 25357 & 25366 & TTTT & 2 & Large subunit ribosomal RNA \\
\hline 73 & P0000051 & Potential SSR & PentaSSR & 26181 & 26190 & CATTT & 2 & (Intron)large subunit ribosomal RNA \\
\hline 74 & P0000052 & Potential SSR & PentaSSR & 27224 & 27233 & ATTTC & 2 & Large subunit ribosomal RNA \\
\hline 75 & P0000053 & Potential SSR & PentaSSR & 27700 & 27709 & TTAAG & 2 & NAD6 \\
\hline 76 & P0000054 & Potential SSR & PentaSSR & 27844 & 27853 & ATAAT & 2 & NAD6 \\
\hline 77 & P0000055 & Potential SSR & PentaSSR & 28013 & 28022 & TAAAA & 2 & NAD6 \\
\hline 78 & P0000056 & Potential SSR & PentaSSR & 29119 & 29128 & TCCCC & 2 & \\
\hline 79 & P0000057 & Potential SSR & PentaSSR & 29273 & 29282 & CAGTA & 2 & $\mathrm{COX} 3$ \\
\hline 80 & P0000058 & Potential SSR & PentaSSR & 29973 & 29982 & TGAT & 2 & $\begin{array}{l}\text { Intron-encoded endonuclease aI1, } \\
\text { (Intron) COX3 }\end{array}$ \\
\hline 81 & P0000059 & Potential SSR & PentaSSR & 30860 & 30869 & AGTG & 2 & (Intron)COX3 \\
\hline 82 & P0000060 & Potential SSR & PentaSSR & 32618 & 32627 & TCCCC & 2 & \\
\hline 83 & P0000061 & Potential SSR & PentaSSR & 33397 & 33406 & TAAAT & 2 & \\
\hline 84 & P0000062 & Potential SSR & PentaSSR & 33416 & 33425 & ATGGT & 2 & \\
\hline 85 & P0000063 & Potential SSR & PentaSSR & 33916 & 33925 & AGAGA & 2 & \\
\hline 86 & P0000064 & Potential SSR & PentaSSR & 34842 & 34851 & AATT & 2 & Small subunit ribosomal RNA \\
\hline 87 & P0000065 & Potential SSR & PentaSSR & 36680 & 36689 & TTAAA & 2 & (Intron)ATP synthase F0 subunit a \\
\hline 88 & P0000066 & Potential SSR & PentaSSR & 36993 & 37002 & TTAAA & 2 & (Intron)ATP synthase F0 subunit a \\
\hline 89 & P0000067 & Potential SSR & PentaSSR & 37021 & 37030 & ATTTT & 2 & (Intron)ATP synthase F0 subunit a \\
\hline 90 & P0000068 & Potential SSR & PentaSSR & 37070 & 37079 & AAGGA & 2 & (Intron)ATP synthase F0 subunit a \\
\hline 91 & P0000069 & Potential SSR & PentaSSR & 37736 & 37745 & ATTTG & 2 & ATP synthase F0 subunit a \\
\hline 92 & P0000070 & Potential SSR & PentaSSR & 38246 & 38255 & TATTT & 2 & \\
\hline 93 & P0000071 & Potential SSR & PentaSSR & 38820 & 38829 & ACAAT & 2 & NAD4 \\
\hline 94 & P0000072 & Potential SSR & PentaSSR & 38940 & 38949 & ATAAA & 2 & NAD4 \\
\hline
\end{tabular}


TABle 7: Continued.

\begin{tabular}{|c|c|c|c|c|c|c|c|c|}
\hline No. & Name & SSR type & Type & Start & End & $\begin{array}{c}\text { Unit } \\
\text { sequence }\end{array}$ & $\begin{array}{l}\text { Repeat } \\
\text { number }\end{array}$ & Genes \\
\hline 95 & P0000073 & Potential SSR & PentaSSR & 40209 & 40218 & TTCAG & 2 & NAD1 \\
\hline 96 & P0000074 & Potential SSR & PentaSSR & 40498 & 40507 & AATAC & 2 & NAD1 \\
\hline 97 & P0000075 & Potential SSR & PentaSSR & 40724 & 40733 & GTTA & 2 & (Intron)NAD1 \\
\hline 98 & P0000076 & Potential SSR & PentaSSR & 41115 & 41124 & AATGG & 2 & (Intron)NAD1 \\
\hline 99 & P0000077 & Potential SSR & PentaSSR & 41463 & 41472 & AATAT & 2 & NAD1 \\
\hline 100 & P0000078 & Potential SSR & PentaSSR & 41867 & 41876 & TACAA & 2 & (Intron)NAD1 \\
\hline 101 & P0000079 & Potential SSR & PentaSSR & 41973 & 41982 & ATATT & 2 & NAD1 \\
\hline 102 & P0000080 & Potential SSR & PentaSSR & 42415 & 42424 & TAGTT & 2 & (Intron)NAD1 \\
\hline 103 & P0000081 & Potential SSR & PentaSSR & 43163 & 43172 & TACAC & 2 & (Intron)COX1 \\
\hline 104 & P0000082 & Potential SSR & PentaSSR & 43808 & 43817 & TATTT & 2 & $\begin{array}{l}\text { LAGLIDADG endonuclease } \\
\text { (QPC56057.1), (Intron)COX1 }\end{array}$ \\
\hline 105 & P0000083 & Potential SSR & PentaSSR & 44007 & 44016 & AATTT & 2 & $\begin{array}{l}\text { LAGLIDADG endonuclease } \\
\text { (QPC56057.1), (Intron)COX1 }\end{array}$ \\
\hline 106 & P0000084 & Potential SSR & PentaSSR & 44079 & 44088 & ATAT & 2 & $\begin{array}{l}\text { LAGLIDADG endonuclease } \\
\text { (QPC56057.1), (Intron)COX1 }\end{array}$ \\
\hline 107 & P0000085 & Potential SSR & PentaSSR & 44160 & 44169 & TTATA & 2 & $\begin{array}{l}\text { LAGLIDADG endonuclease } \\
\text { (QPC56057.1), (Intron)COX1 }\end{array}$ \\
\hline 108 & P0000086 & Potential SSR & PentaSSR & 44359 & 44368 & TAATT & 2 & $\begin{array}{l}\text { LAGLIDADG endonuclease } \\
\text { (QPC56057.1), (Intron)COX1 }\end{array}$ \\
\hline 109 & P0000087 & Potential SSR & PentaSSR & 47717 & 47726 & TGTTT & 2 & $\begin{array}{l}\text { LAGLIDADG endonuclease } \\
\text { (QPC56054.1), (Intron)COX1 }\end{array}$ \\
\hline 110 & P0000088 & Potential SSR & PentaSSR & 48411 & 48420 & ATATA & 2 & (Intron)COX1 \\
\hline 111 & P0000089 & Potential SSR & PentaSSR & 48965 & 48974 & TATAT & 2 & $\begin{array}{l}\text { LAGLIDADG endonuclease } \\
\text { (QPC56060.1), (Intron)COX1 }\end{array}$ \\
\hline 112 & P0000090 & Potential SSR & PentaSSR & 49852 & 49861 & TATTT & 2 & $\begin{array}{l}\text { LAGLIDADG endonuclease } \\
\text { (QPC56055.1), (Intron)COX1 }\end{array}$ \\
\hline 113 & P0000091 & Potential SSR & PentaSSR & 50038 & 50047 & ATAAA & 2 & $\begin{array}{l}\text { LAGLIDADG endonuclease } \\
\text { (QPC56055.1), (Intron)COX1 }\end{array}$ \\
\hline 114 & P0000092 & Potential SSR & PentaSSR & 50572 & 50581 & AAATA & 2 & $\begin{array}{l}\text { LAGLIDADG endonuclease } \\
\text { (QPC56055.1), (Intron)COX1 }\end{array}$ \\
\hline 115 & P0000093 & Potential SSR & PentaSSR & 50686 & 50695 & CATAG & 2 & $\begin{array}{l}\text { LAGLIDADG endonuclease } \\
\text { (QPC56055.1), (Intron)COX1 }\end{array}$ \\
\hline 116 & P0000094 & Potential SSR & PentaSSR & 50901 & 50910 & TATTT & 2 & $\begin{array}{l}\text { LAGLIDADG endonuclease } \\
\text { (QPC56059.1), (Intron)COX1 }\end{array}$ \\
\hline 117 & P0000095 & Potential SSR & PentaSSR & 52105 & 52114 & ATAG & 2 & (Intron)COX1 \\
\hline 118 & P0000096 & Potential SSR & PentaSSR & 52165 & 52174 & TATTT & 2 & (Intron)COX1 \\
\hline 119 & P0000097 & Potential SSR & PentaSSR & 53150 & 53159 & TTTAC & 2 & (Intron)COX1 \\
\hline 120 & P0000098 & Potential SSR & PentaSSR & 53214 & 53223 & ATAT & 2 & (Intron)COX1 \\
\hline 121 & P0000099 & Potential SSR & PentaSSR & 53261 & 53270 & TTATA & 2 & (Intron)COX1 \\
\hline 122 & P0000100 & Potential SSR & PentaSSR & 53525 & 53534 & ATATT & 2 & (Intron)COX1 \\
\hline 123 & P0000101 & Potential SSR & PentaSSR & 55085 & 55094 & ATAT & 2 & COX1 \\
\hline 124 & H0000001 & Potential SSR & HexaSSR & 1409 & 1420 & ATTTAG & 2 & (Intron)Cob \\
\hline 125 & H0000002 & Potential SSR & HexaSSR & 1544 & 1555 & GAATTA & 2 & (Intron)Cob \\
\hline 126 & H0000003 & Potential SSR & HexaSSR & 1819 & 1830 & TTAATC & 2 & (Intron)Cob \\
\hline 127 & H0000004 & Potential SSR & HexaSSR & 2353 & 2364 & ATTTT & 2 & (Intron)Cob \\
\hline 128 & H0000005 & Normal SSR & HexaSSR & 2548 & 2565 & AAATAT & 3 & Cob \\
\hline 129 & H0000006 & Potential SSR & HexaSSR & 2996 & 3007 & TTTTTA & 2 & (Intron)Cob \\
\hline 130 & H0000008 & Potential SSR & HexaSSR & 5935 & 5946 & TTTATT & 2 & (Intron)Cob \\
\hline 131 & H0000009 & Potential SSR & HexaSSR & 6512 & 6523 & TAAATC & 2 & (Intron)Cob \\
\hline
\end{tabular}


TABle 7: Continued.

\begin{tabular}{|c|c|c|c|c|c|c|c|c|}
\hline No. & Name & SSR type & Type & Start & End & $\begin{array}{c}\text { Unit } \\
\text { sequence }\end{array}$ & $\begin{array}{l}\text { Repeat } \\
\text { number }\end{array}$ & Genes \\
\hline 132 & H0000011 & Potential SSR & HexaSSR & 7506 & 7517 & GATTA & 2 & $\begin{array}{c}\text { LAGLIDADG/HNH } \\
\text { endonuclease, (Intron)Cob }\end{array}$ \\
\hline 133 & H0000012 & Potential SSR & HexaSSR & 8965 & 8976 & AACTA & 2 & NAD5 \\
\hline 134 & H0000013 & Potential SSR & HexaSSR & 9972 & 9983 & ATCCC & 2 & NAD5 \\
\hline 135 & H0000014 & Potential SSR & HexaSSR & 12348 & 12359 & TAAAT & 2 & (Intron) $\mathrm{COX} 2$ \\
\hline 136 & H0000015 & Potential SSR & HexaSSR & 12475 & 12486 & AAAGT & 2 & (Intron) $\mathrm{COX} 2$ \\
\hline 137 & H0000016 & Potential SSR & HexaSSR & 13479 & 13490 & ATTTA & 2 & (Intron)COX2 \\
\hline 138 & H0000017 & Potential SSR & HexaSSR & 17949 & 17960 & GTTAAT & 2 & NAD2 \\
\hline 139 & H0000018 & Potential SSR & HexaSSR & 17975 & 17986 & TAAAAA & 2 & NAD2 \\
\hline 140 & H0000019 & Potential SSR & HexaSSR & 19353 & 19364 & TAATAC & 2 & \\
\hline 141 & H0000020 & Potential SSR & HexaSSR & 21110 & 21121 & TTTTAA & 2 & $\begin{array}{c}\text { RPS3, (Intron)large } \\
\text { subunit ribosomal RNA }\end{array}$ \\
\hline 142 & H0000023 & Potential SSR & HexaSSR & 22403 & 22414 & TATGCC & 2 & Large subunit ribosomal RNA \\
\hline 143 & H0000024 & Potential SSR & HexaSSR & 23824 & 23835 & TCCGCA & 2 & Large subunit ribosomal RNA \\
\hline 144 & H0000025 & Potential SSR & HexaSSR & 24585 & 24596 & GAACT & 2 & Large subunit ribosomal RNA \\
\hline 145 & H0000026 & Potential SSR & HexaSSR & 26510 & 26521 & AAATA & 2 & (Intron)large subunit ribosomal RNA \\
\hline 146 & H0000027 & Potential SSR & HexaSSR & 27040 & 27051 & TATTTT & 2 & Large subunit ribosomal RNA \\
\hline 147 & H0000028 & Potential SSR & HexaSSR & 27669 & 27680 & TTTAT & 2 & NAD6 \\
\hline 148 & H0000029 & Potential SSR & HexaSSR & 28253 & 28264 & TATTAA & 2 & NAD6 \\
\hline 149 & H0000030 & Potential SSR & HexaSSR & 31008 & 31019 & TCTGA & 2 & (Intron) $\mathrm{COX} 3$ \\
\hline 150 & H0000031 & Potential SSR & HexaSSR & 34196 & 34207 & TAGTT & 2 & \\
\hline 151 & H0000032 & Potential SSR & HexaSSR & 36802 & 36813 & GTGTA & 2 & (Intron)ATP synthase F0 subunit a \\
\hline 152 & H0000034 & Potential SSR & HexaSSR & 37885 & 37896 & AGATAA & 2 & ATP synthase F0 subunit a \\
\hline 153 & H0000035 & Potential SSR & HexaSSR & 41287 & 41298 & ATTTAA & 2 & NAD1 \\
\hline 154 & H0000036 & Potential SSR & HexaSSR & 44425 & 44436 & TCCATC & 2 & $\begin{array}{l}\text { LAGLIDADG endonuclease } \\
\text { (QPC56057.1), (Intron)COX1 }\end{array}$ \\
\hline 155 & H0000037 & Potential SSR & HexaSSR & 45779 & 45790 & TCCATC & 2 & $\begin{array}{l}\text { LAGLIDADG endonuclease } \\
\text { (QPC56058.1), (Intron)COX1 }\end{array}$ \\
\hline 156 & H0000038 & Potential SSR & HexaSSR & 46175 & 46186 & TATTTA & 2 & (Intron)COX1 \\
\hline 157 & H0000039 & Potential SSR & HexaSSR & 46345 & 46356 & TTATT & 2 & (Intron)COX1 \\
\hline 158 & H0000040 & Potential SSR & HexaSSR & 46609 & 46620 & TTAATA & 2 & (Intron)COX1 \\
\hline 159 & H0000041 & Potential SSR & HexaSSR & 47358 & 47369 & ATAAAC & 2 & $\begin{array}{l}\text { LAGLIDADG endonuclease } \\
\text { (QPC56054.1), (Intron)COX1 }\end{array}$ \\
\hline 160 & H0000042 & Potential SSR & HexaSSR & 50889 & 50900 & TTTTAA & 2 & $\begin{array}{l}\text { LAGLIDADG endonuclease } \\
\text { (QPC56059.1), (Intron)COX1 }\end{array}$ \\
\hline 161 & H0000043 & Potential SSR & HexaSSR & 53483 & 53494 & СТTAT & 2 & (Intron)COX1 \\
\hline 162 & H0000044 & Potential SSR & HexaSSR & 54105 & 54116 & TTACCC & 2 & (Intron)COX1 \\
\hline 163 & H0000045 & Potential SSR & HexaSSR & 55364 & 55375 & TTCT & 2 & \\
\hline 164 & cHp0000001 & $\begin{array}{l}\text { Extended } \\
\text { SSR }\end{array}$ & HeptaSSR & 898 & 911 & AATTATA & 2 & (Intron)Cob \\
\hline 165 & cHp0000002 & $\begin{array}{l}\text { Extended } \\
\text { SSR }\end{array}$ & HeptaSSR & 13979 & 13992 & AATAATA & 2 & \\
\hline 166 & cHp0000003 & $\begin{array}{l}\text { Extended } \\
\text { SSR }\end{array}$ & HeptaSSR & 15909 & 15922 & GGTATTT & 2 & $\begin{array}{l}\text { Hypothetical protein, } \\
\text { (Intron)NAD2 }\end{array}$ \\
\hline 167 & cHp0000005 & $\begin{array}{l}\text { Extended } \\
\text { SSR }\end{array}$ & HeptaSSR & 34242 & 34255 & TTATAA & 2 & Small subunit ribosomal RNA \\
\hline 168 & cHp0000006 & $\begin{array}{l}\text { Extended } \\
\text { SSR }\end{array}$ & HeptaSSR & 44930 & 44943 & ATTATT & 2 & (Intron)COX1 \\
\hline 169 & O0000001 & $\begin{array}{l}\text { Extended } \\
\text { SSR }\end{array}$ & OctaSSR & 40662 & 40677 & TTCATAT & 2 & (Intron)NAD1 \\
\hline
\end{tabular}




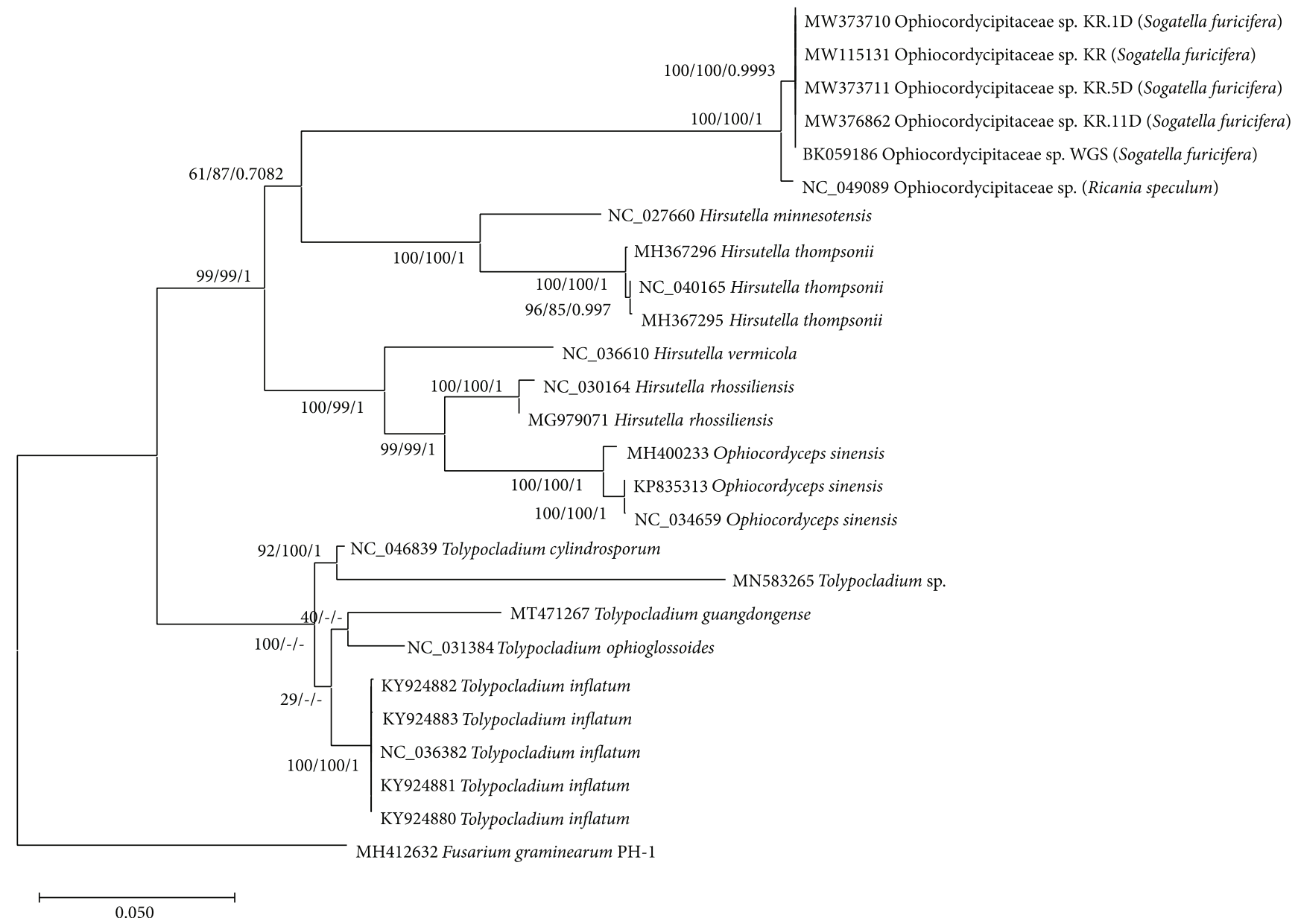

FIGURE 5: Phylogenetic trees of 26 fungal complete mitogenomes in Ophiocordycipitaceae. Neighbor-joining (bootstrap repeat is 10,000) and maximum-Likelihood (bootstrap repeat is 1000) phylogenetic trees as well as Bayesian inference tree $(1,100,000$ generations) of 26 fungal mitogenomes of Ophiocordycipitaceae. Phylogenetic tree was displayed based on the maximum-likelihood tree. The numbers above branches indicate bootstrap support values of maximum-likelihood and neighbor-joining phylogenetic trees and posterior possibility value of Bayesian inference tree, respectively. Scientific names inside the parenthesis indicate those of host species.

The length of the identified SSRs is relatively short (a maximum length of $18 \mathrm{bp}$; Figure $4(\mathrm{a})$ ) in comparison to those of other fungal species in the same family: Ophiocordyceps sinensis (up to $24 \mathrm{bp}$ ) [123], as well as fungal species in the other families, such as Pestalotiopsis fici (up to $45 \mathrm{bp}$ ) [124]. Moreover, the maximum length of SSRs identified from the mitogenome of $R$. speculum (NC_049089) [19] was $18 \mathrm{bp}$, suggesting that this short SSR length can be linked to the evolution of endosymbiont mitogenomes.

Out of 191 normal SSRs, extended SSRs, and potential SSRs, 84 (43.98\%) are located in the genic region (genic and intronic ORF categories in Figure 4(b); Table 7). The intronic ORF position indicates the location of the PCGs placed at the introns of other PCGs, most of which are LAGLIDADG endonucleases (Table 2). Nearly half of the SSRs are in PCGs, which are conserved in comparison to intron and intergenic regions, indicating that these SSRs can be utilized for distinguishing species level or even higher rank. In the intergenic region, there were 61 SSRs (31.94\%), and in comparison, only 24 SSRs (12.57\%) in the intergenic region (Figure 4(b);
Table 7). These SSRs are located in relatively nonconserved regions in comparison to PCG regions, suggesting that these SSRs can be used to distinguish intraspecific levels, such as population or geographical origins. Once more endosymbiont mitogenomes are available in the near future, these SSRs can be evaluated for their use in identification of species and their geographical origin as well as evolutionary history of their mitogenomes.

In the genic region, 84 SSRs were distributed in 24 different genes consisting of 21 PCGs, 2 rRNAs, and 1 tRNA (Figure 4(c); Table 7). The large subunit RNA contained the most SSRs and the genes COX1, COX3, NAD3, two LAGLIDADG endonucleases, intron-encoded nuclease aIl, hypothetical protein, and tRNA-Glu contained the fewest (Figure 4(c); Table 7). Considering the length of these genes, some, including large submit RNA, NAD2, LAGLIDADG endonuclease (QPC56057.1), NAD1, NAD6, ATP synthase F0 subunit a, and LAGLIDADG/HNH endonuclease, displayed a relatively large number of SSRs (Figure 4(c); Table 7). Meanwhile, the remaining genes have a relatively 
low number of SSRs. This inequality of SSR distribution in PCGs can be another useful characteristic for developing efficient molecular markers. In addition, SSRs in PCGs are known to affect the functions of those PCGs especially for adaptation to environmental factors in fungi [125-127], suggesting that these SSRs can also affect the functions of mitochondrial PCGs.

\subsection{Phylogenetic Analysis of 25 Fungal Mitogenomes of} Ophiocordycipitaceae. We constructed bootstrapped maximum-likelihood (ML) and Bayesian inference (BI) phylogenetic trees using 26 fungal mitogenomes consisting of 5 mitogenomes used in this study, 25 mitogenomes in the Ophiocordycipitaceae family, and 1 outgroup species (Fusarium graminearum) [128]. Due to the incomplete annotation of the Ophiocordyceps sinensis fungal mitogenome (KP835313), five PCGs, NAD5, COB, COX1, NAD1, and $N A D 4$, containing introns are not correctly annotated. Only five conserved PCGs, ATP8, COX2, NAD2, NAD3, and $N A D 4 L$, were selected and aligned individually. Subsequently, this alignment was concatenated to construct three phylogenetic trees.

Five fungal endosymbiont mitogenomes of WBPH were well clustered with another fungal symbiont mitogenome of R. speculum (NC_049089) [19] with high supportive values (Figure 5). This indicates taxonomic similarity between the $R$. speculum endosymbiont and the five WBPH endosymbionts, suggesting that other fungal endosymbionts may also be independently clustered with other fungal species in the sample family, Ophiocordycipitaceae. In terms of evolution, it can be explained by the two hypotheses: (i) independent evolution once this endosymbiont entered the host insect species or (ii) independent taxonomic groups of Ophiocordycipitaceae entering into the host insect species multiple times during evolution. To determine which hypothesis is more likely, we would need more endosymbiont mitogenomes from various host insect species of infraorder Fulgoromorpha and suborder Auchenorrhyncha as well as mitogenomes from neighboring noninsect endosymbiont fungal species.

Four fungal species used to investigate intraspecific variations in mitogenomes, Hirsutella thompsonii, Hirsutella rhossiliensis, Ophiocordyceps sinensis, and Tolypocladium inflatum, also display rigid clades covering all mitogenomes of each species with high supportive values (Figure 5). Three mitogenomes of Ophiocordyceps sinensis were clustered with the longest branch length among the four species, of which Hirsutella thompsonii had the second longest (Figure 5). These branch lengths were not proportional to the ratio of SNPs and INDELs (Table 4). The topology of the Tolypocladium genus in the trees was not congruent between the ML and BI trees with low bootstrap values (Figure 5), indicating that additional conserved gene sequences are required to resolve this clade properly.

\section{Conclusions}

We successfully elucidated the five complete mitogenomes of the fungal endosymbiont of WBPH from various sources of
NGS raw reads obtained from WBPH samples. These five complete mitogenomes show common and their own characteristics in comparison to the previously elucidated complete mitogenome of the $R$. japonica fungal endosymbiont [19]. There were fewer intraspecific variations in the five WBPH endosymbiont mitogenomes in comparison to those identified from the four Ophiocordycipitaceae fungal species, Ophiocordyceps sinensis, Hirsutella thompsonii, Hirsutella rhossiliensis, and Tolypocladium inflatum. This can be explained by the narrow geographical distribution and/or genetic background and the low selection pressures of endosymbionts. We identified 191 SSRs were from each WBPH fungal symbiont complete mitogenomes, except for the WBPH_KR.1D mitogenome, which presented an additional SSR. These SSRs are relatively short in length (a maximum length of $18 \mathrm{bp}$ ) compared to those of other fungal mitogenomes. Nearly half of the SSRs are in the genic region, suggesting that these SSRs may be more conserved and they may affect the functionality of PCGs. Based on the phylogenetic trees of 5 conserved PCGs of 26 fungal mitogenomes, including one outgroup species, WBPH fungal endosymbiont mitogenomes were clustered with that of $R$. speculum with high supportive values. This suggests that these insect-hosted fungal endosymbionts have been evolved independently from the other fungal species in the Ophiocordycipitaceae family. Owing to the advantages of NGS raw reads, which can detect sequences from unknown or unexpected organisms [12, 1937], we successfully identified the complete mitogenomes of WBPH fungal endosymbionts within the NGS raw reads, suggesting that we can understand their phylogenetic positions of fungal symbiont with high resolution without the need to isolate the symbiont from the host. Furthermore, our study shows that NGS raw reads of insects generated in the future can be used to pinpoint further fungal endosymbionts that have previously been difficult to identify. This method could provide novel insights into their phylogenetic positions as well as interactions with their host species.

\section{Data Availability}

Mitochondrial genome sequence used in this study can be accessed via accession numbers MW115131, MW373710, MW373711, MW376862, and BK059186 in the NCBI GenBank.

\section{Conflicts of Interest}

The authors declare that they have no competing interests.

\section{Acknowledgments}

This work was carried out with the support of "Cooperative Research Program for Agriculture Science \& Technology Development (project title: Population Analysis and Development Technology to Predict Change of White Back Planthopper Population, Project No. PJ01386402)," Rural Development Administration, Republic of Korea. We also would like to thank Editage (http://www.editage.co.kr) for English language editing. 


\section{References}

[1] J. Evans, "A natural classification of leaf-hoppers (Jassoidea, Homoptera) part 1. External morphology and systematic position," Transactions of the Royal Entomological Society of London., vol. 96, no. 3, pp. 47-60, 1946.

[2] N. Song, A.-P. Liang, and C.-P. Bu, “A molecular phylogeny of Hemiptera inferred from mitochondrial genome sequences," PLoS One, vol. 7, no. 11, article e48778, 2012.

[3] R. Kisimoto and K. Sogawa, "Migration of the brown planthopper Nilaparvata lugens and the white-backed planthopper Sogatella furcifera in East Asia: the role of weather and climate," Insect migration: tracking resources through space and time., pp. 67-92, 1995, https://www .cambridge.org/core/books/insect-migration/migration-ofthe-brown-planthopper-nilaparvata-lugens-and-thewhitebacked-planthopper-sogatella-furcifera-in-east-asia$\mathrm{th}$ e - r o l e - of - w e a t h e r - a n d - cli m a t e / 761D90923CD10E1CF0E33C5D72AD28D0.

[4] W. T. Garrood, C. T. Zimmer, K. J. Gorman, R. Nauen, C. Bass, and T. G. Davies, "Field-evolved resistance to imidacloprid and ethiprole in populations of brown planthopper Nilaparvata lugens collected from across South and East Asia," Pest Management Science, vol. 72, no. 1, pp. 140-149, 2016.

[5] A. Otuka, M. Matsumura, S. Sanada-Morimura et al., "The 2008 overseas mass migration of the small brown planthopper, Laodelphax striatellus, and subsequent outbreak of rice stripe disease in western Japan," Applied Entomology and Zoology, vol. 45, no. 2, pp. 259-266, 2010.

[6] J. Cheng, "Rice planthopper problems and relevant causes in China," Planthoppers: new threats to the sustainability of intensive rice production systems in Asia., vol. 157, p. 178, 2009.

[7] R. Kisimoto, 12. Long distance migration of planthoppers, Sogatella furcifera A, ND Nilaparvata lugens, 1971.

[8] J. Park, J.-H. An, Y. Kim, D. Kim, B.-G. Yang, and T. Kim, "Database of National Species List of Korea: the taxonomical systematics platform for managing scientific names of Korean native species," Journal of Species Research, vol. 9, no. 3, pp. 233-246, 2020.

[9] Z. Khan and R. Saxena, "Behavioral and physiological responses of Sogatella furcifera (Homoptera: Delphacidae) to selected resistant and susceptible rice cultivars," Journal of Economic Entomology, vol. 78, no. 6, pp. 1280-1286, 1985.

[10] K.-J. Zhang, W.-C. Zhu, X. Rong, J. Liu, X.-L. Ding, and X.Y. Hong, "The complete mitochondrial genome sequence of Sogatella furcifera (Horváth) and a comparative mitogenomic analysis of three predominant rice planthoppers," Gene, vol. 533, no. 1, pp. 100-109, 2014.

[11] L. Wang, N. Tang, X. Gao et al., "Genome sequence of a rice pest, the white-backed planthopper (Sogatella furcifera)," Gigascience, vol. 6, no. 1, article giw004, pp. 1-9, 2017.

[12] Z. Zeng, Y. Fu, D. Guo, Y. Wu, O. E. Ajayi, and Q. Wu, "Bacterial endosymbiont Cardinium cSfur genome sequence provides insights for understanding the symbiotic relationship in Sogatella furcifera host," BMC Genomics, vol. 19, no. 1, p. 688, 2018.

[13] J. H. Werren, L. Baldo, and M. E. Clark, "Wolbachia: master manipulators of invertebrate biology," Nature Reviews. Microbiology, vol. 6, no. 10, pp. 741-751, 2008.
[14] Y. Nakamura, F. Yukuhiro, M. Matsumura, and H. Noda, "Cytoplasmic incompatibility involving Cardinium and Wolbachia in the white-backed planthopper Sogatella furcifera (Hemiptera: Delphacidae)," Applied Entomology and Zoology, vol. 47, no. 3, pp. 273-283, 2012.

[15] Y. Hongoh and H. Ishikawa, "Evolutionary studies on uricases of fungal endosymbionts of aphids and planthoppers," Journal of Molecular Evolution, vol. 51, no. 3, pp. 265-277, 2000.

[16] S. Dong, K. Pang, X. Bai, X. Yu, and P. Hao, "Identification of two species of yeast-like symbiotes in the brown Planthopper, Nilaparvata lugens, Nilaparvata lugens," Current Microbiology, vol. 62, no. 4, pp. 1133-1138, 2011.

[17] C. C. Chen, L. L. Cheng, and R. F. Hou, "Studies on the intracellular yeast-like symbiote in the Brown Planthopper, Nilaparvata lugens StÅl: II. Effects of antibiotics and elevated temperature on the symbiotes and their host," Zeitschrift für Angewandte Entomologie, vol. 92, no. 1-5, pp. 440-449, 1981.

[18] T. Sasaki, M. Kawamura, and H. Ishikawa, "Nitrogen recycling in the brown planthopper, Nilaparvata lugens: involvement of yeast-like endosymbionts in uric acid metabolism," Journal of Insect Physiology, vol. 42, no. 2, pp. 125-129, 1996.

[19] J. Park, H. Xi, J. Park, and W. Lee, “The complete mitochondrial genome of fungal endosymbiont, Ophiocordycipitaceae sp., isolated from Ricania speculum (Hemiptera: Ricaniidae)," Mitochondrial DNA Part B., vol. 5, no. 2, pp. 1888-1889, 2020.

[20] J. Park, H. Xi, J. Park, S. J. Nam, and Y.-D. Lee, “Complete genome sequence of the Blochmannia Endosymbiont of Camponotus nipponensis," Microbiology Resource Announcements, vol. 9, no. 29, pp. e00703-e00720, 2020.

[21] J. Park, H. Xi, Y. Kim, S. Nam, and K.-I. Heo, “The complete mitochondrial genome of new species candidate ofRosa rugosa(Rosaceae)," Mitochondrial DNA Part B., vol. 5, no. 3, pp. 3435-3437, 2020.

[22] H. Lee, J. Park, H. Xi et al., "The complete mitochondrial genome of Ricania speculum (Walker, 1851) (Hemiptera: Ricaniidae): investigation of intraspecific variations on mitochondrial genome," Mitochondrial DNA Part B., vol. 5, no. 3, pp. 3796-3798, 2020.

[23] Y. Bae, J. Park, and W. Lee, "The complete mitochondrial genome of Aphis gossypii Glover, 1877 (Hemiptera: Aphididae) isolated from Plantago asiatica in Korea," Mitochondrial DNA Part B., vol. 5, no. 3, pp. 2878-2880, 2020.

[24] J. Park, Y. Kim, H. Xi, J. Park, and W. Lee, “The complete mitochondrial genome of Rhopalosiphum nymphaeae (Linnaeus, 1761) (Hemiptera: Aphididae)," Mitochondrial DNA Part B., vol. 5, no. 2, pp. 1613-1615, 2020.

[25] Y. Kim, K.-I. Heo, S. Nam, H. Xi, S. Lee, and J. Park, “The complete chloroplast genome of candidate new species from Rosa rugosa in Korea (Rosaceae)," Mitochondrial DNA Part B., vol. 4, no. 2, pp. 2433-2435, 2019.

[26] J. Park, Y. Kim, H. Xi, W. Kwon, and M. Kwon, "The complete chloroplast and mitochondrial genomes of Hyunsasi tree, Populus alba x Populus glandulosa (Salicaceae)," Mitochondrial DNA Part B., vol. 4, no. 2, pp. 2521-2522, 2019.

[27] J. Park, Y. Kim, W. Kwon, H. Xi, and M. Kwon, "The complete chloroplast genome of tulip tree, Liriodendron tulifipera L. (Magnoliaceae): investigation of intra-species chloroplast variations," Mitochondrial DNA Part B., vol. 4, no. 2, pp. 2523-2524, 2019. 
[28] J. Park, Y. Kim, and M. Kwon, “The complete mitochondrial genome of tulip tree, Liriodendron tulipifera L. (Magnoliaceae): intra-species variations on mitochondrial genome," Mitochondrial DNA Part B., vol. 4, no. 1, pp. 1308-1309, 2019.

[29] W. Kwon, Y. Kim, and J. Park, "The complete chloroplast genome of Korean Marchantia polymorpha subsp. ruderalis Bischl. \& Boisselier: low genetic diversity between Korea and Japan," Mitochondrial DNA Part B., vol. 4, no. 1, pp. 959-960, 2019.

[30] W. Kwon, Y. Kim, and J. Park, "The complete mitochondrial genome of Korean Marchantia polymorpha subsp. ruderalis Bischl. \& Boisselier: inverted repeats on mitochondrial genome between Korean and Japanese isolates," Mitochondrial DNA Part B., vol. 4, no. 1, pp. 769-770, 2019.

[31] W. Kwon, Y. Kim, and J. Park, "The complete chloroplast genome sequence of Dumortiera hirsuta (Sw.) Nees (Marchantiophyta, Dumortieraceae)," Mitochondrial DNA Part B., vol. 4, no. 1, pp. 318-319, 2019.

[32] W. Kwon, Y. Kim, and J. Park, “The complete mitochondrial genome of Dumortiera hirsuta (Sw.) Nees (Dumortieraceae, Marchantiophyta)," Mitochondrial DNA Part B., vol. 4, no. 1, pp. 1586-1587, 2019.

[33] J. Min, W. Kwon, H. Xi, and J. Park, "The complete mitochondrial genome of Riccia fluitans L. (Ricciaceae, Marchantiophyta): investigation of intraspecific variations on mitochondrial genomes of $R$. fluitans," Mitochondrial DNA Part B., vol. 5, no. 2, pp. 1220-1222, 2020.

[34] W. Kwon, J. Min, H. Xi, and J. Park, “The complete chloroplast genome of Riccia fluitans L. (Ricciaceae, Marchantiophyta)," Mitochondrial DNA Part B., vol. 4, no. 1, pp. 18951896, 2019.

[35] S. S. Choi, W. Kwon, and J. Park, "The complete chloroplast genome of Wiesnerella denudata (Mitt.) Steph. (Wiesnerellaceae, Marchantiophyta)," Mitochondrial DNA Part B., vol. 5, no. 3, pp. 3124-3126, 2020.

[36] S. S. Choi, J. Min, W. Kwon, and J. Park, "The complete mitochondrial genome of Wiesnerella denudata (Mitt.) Steph. (Wiesnerellaceae, Marchantiophyta): large number of intraspecific variations on mitochondrial genomes of $W$. denudata," Mitochondrial DNA Part B., vol. 5, no. 3, pp. 3351-3353, 2020.

[37] J. Park, H. Xi, and J. Park, "Complete genome sequence of a Blochmannia Endosymbiont of Colobopsis nipponica," Microbiology Resource Announcements., vol. 10, no. 17, p. e01195, 2021.

[38] D. R. Zerbino and E. Birney, "Velvet: algorithms for de novo short read assembly using de Bruijn graphs," Genome Research, vol. 18, no. 5, pp. 821-829, 2008.

[39] A. M. Bolger, M. Lohse, and B. Usadel, "Trimmomatic: a flexible trimmer for Illumina sequence data," Bioinformatics, vol. 30, no. 15, pp. 2114-2120, 2014.

[40] Q.-Y. Zhao, Y. Wang, Y.-M. Kong, D. Luo, X. Li, and P. Hao, "Optimizing de novo transcriptome assembly from shortread RNA-Seq data: a comparative study," BMC Bioinformatics, vol. 12, no. 14, p. S2, 2011.

[41] H. Li, B. Handsaker, A. Wysoker et al., "The sequence alignment/map format and SAMtools," Bioinformatics, vol. 25, no. 16, pp. 2078-2079, 2009.

[42] H. Li, Aligning sequence reads, clone sequences and assembly contigs with BWA-MEM, 2013, arXiv preprint arXiv:13033997.
[43] Y.-D. Lee, J. Lee, D.-S. Kim et al., “The complete mitochondrial genome of Hipparchia autonoe (Esper, 1783) (Lepidoptera: Nymphalidae): investigation of intraspecific variations on mitochondrial genome," Mitochondrial DNA Part B., vol. 5, no. 2, pp. 1542-1544, 2020.

[44] J. Lee, J. Park, H. Xi, and J. Park, "Comprehensive analyses of the complete mitochondrial genome of Figulus binodulus (Coleoptera: Lucanidae)," Journal of Insect Science, vol. 20, no. 5, p. 10, 2020.

[45] N. J. Choi, B.-C. Lee, J. Park, and J. Park, "The complete mitochondrial genome of Nilaparvata lugens (Stål, 1854) captured in Guangxi province, China (Hemiptera: Delphacidae): identification of the origin of $N$. lugens migrated to Korea," Mitochondrial DNA Part B., vol. 5, no. 2, pp. 1960-1961, 2020.

[46] B. Y. Seo, G.-S. Lee, J. Park et al., "The complete mitochondrial genome of the fall armyworm, Spodoptera frugiperda Smith, 1797 (Lepidoptera; Noctuidae), firstly collected in Korea," Mitochondrial DNA Part B., vol. 4, no. 2, pp. 39183920, 2019.

[47] B. Y. Seo, J. K. Jung, Y. Ho Koh, and J. Park, “The complete mitochondrial genome of Laodelphax striatellus (Fallén, 1826) (Hemiptera: Delphacidae) collected in a southern part of Korean peninsula," Mitochondrial DNA Part B., vol. 4, no. 2, pp. 2242-2243, 2019.

[48] J. Park, H. Xi, W. Kwon, C.-G. Park, and W. Lee, "The complete mitochondrial genome sequence of Korean Chilo suppressalis (Walker, 1863) (Lepidoptera: Crambidae)," Mitochondrial DNA Part B, vol. 4, no. 1, pp. 850-851, 2019.

[49] J. Park, H. Xi, Y. Kim, J. Park, and W. Lee, "The complete mitochondrial genome of Aphis gossypii Glover, 1877 (Hemiptera: Aphididae) collected in Korean peninsula," Mitochondrial DNA Part B., vol. 4, no. 2, pp. 3007-3009, 2019.

[50] J. Park, J. K. Jung, G.-S. Lee, J. Cho, Y. H. Koh, and B. Y. Seo, "Comparative analyses of Laodelphax striatellus mitochondrial genomes: Insight haplotypes of mitogenomes in three East Asian Countries," in 2019 Fall International Conference of KSAE, Seoul, Korea, 2019.

[51] J. Park, J. K. Jung, Y. Ho Koh, J. Park, and B. Y. Seo, “The complete mitochondrial genome of Laodelphax striatellus (Fallén, 1826) (Hemiptera: Delphacidae) collected in a midWestern part of Korean peninsula," Mitochondrial DNA Part B., vol. 4, no. 2, pp. 2229-2230, 2019.

[52] J. Park, M.-K. Lee, J.-H. Yu, B. Zhu, J.-H. Kim, and K.H. Han, "Complete mitochondrial genome sequence of $<i>$ Aspergillus flavus $</ i>$ SRRC1009: insight of intraspecific variations on $<\mathrm{i}>\mathrm{A}$. flavus $</ \mathrm{i}>$ mitochondrial genomes," Mitochondrial DNA Part B., vol. 5, no. 3, pp. 3585-3587, 2020.

[53] J. Park, M.-K. Lee, J.-H. Yu, J.-H. Kim, and K.-H. Han, “Complete mitochondrial genome sequence of Afla-Guard ${ }^{\circledR}$, commercially available non-toxigenic Aspergillus flavus," Mitochondrial DNA B Resour, vol. 5, no. 3, pp. 3590-3592, 2020.

[54] J. Park, W. Kwon, J.-B. Kim, M.-J. Park, and T.-S. Kim, "Complete mitochondrial genome sequence of lettuce pathogenic fungus, Fusarium oxysporum f. sp. lactucae 09-002," Mitochondrial DNA Part B., vol. 4, no. 2, pp. 3434-3436, 2019.

[55] J. Park, W. Kwon, X. Huang et al., "Complete mitochondrial genome sequence of a xerophilic fungus, Aspergillus pseudoglaucus," Mitochondrial DNA Part B, vol. 4, no. 2, pp. 24222423, 2019. 
[56] W. Kwon, J. Park, J.-B. Kim, M.-J. Park, and T.-S. Kim, "Complete mitochondrial genome sequence of lettuce pathogenic fungus, Fusarium oxysporum f. sp. lactucae 16-086," Mitochondrial DNA Part B., vol. 4, no. 2, pp. 3227-3228, 2019.

[57] J. Park, W. Kwon, and J. Park, "The complete mitochondrial genome of Siberian odorous ant, Dolichoderus sibiricus Emery, 1889 (Hymenoptera: Formicidae)," Mitochondrial DNA Part B., vol. 4, no. 1, pp. 525-526, 2019.

[58] J. Park, W. Kwon, and J. Park, "The complete mitochondrial genome of Cryptopone sauteri Wheeler, WM, 1906 (Hymenoptera: Formicidae)," Mitochondrial DNA Part B., vol. 4, no. 1, pp. 614-615, 2019.

[59] J. Park, H. Xi, and J. Park, "The complete mitochondrial genome of Aphaenogaster famelica (Smith, 1874)(Hymenoptera: Formicidae)," Mitochondrial DNA Part B., vol. 5, no. 1, pp. 492-494, 2020.

[60] J. Park, H. Xi, and J. Park, "The complete mitochondrial genome of Nylanderia flavipes (Smith, 1874)(Hymenoptera: Formicidae)," Mitochondrial DNA Part B., vol. 5, no. 1, pp. 420-421, 2020.

[61] J. Park, H. Xi, and J. Park, "The complete mitochondrial genome of Ochetellus glaber (Mayr, 1862)(Hymenoptera: Formicidae)," Mitochondrial DNA Part B., vol. 5, no. 1, pp. 147-149, 2020.

[62] J. Park, W. Kwon, and J. Park, "The complete mitochondrial genome of Camponotus concavus Kim \& Kim, 1994 (Hymenoptera: Formicidae)," Mitochondrial DNA Part B., vol. 4, no. 1, pp. 1243-1244, 2019.

[63] J. Park, W. Kwon, B. Zhu et al., "Complete mitochondrial genome sequence of the food fermentation fungus, Aspergillus luchuensis," Mitochondrial DNA B Resour, vol. 4, no. 1, pp. 945-946, 2019.

[64] W. Lee, T. Han, J.-H. Lee, K.-J. Hong, and J. Park, “The complete mitochondrial genome of the subterranean termite, Reticulitermes speratus kyushuensis Morimoto, 1968 (Isoptera: Rhinotermitidae)," Mitochondrial DNA Part B., vol. 2, no. 1, pp. 178-179, 2017.

[65] J. Lee, J. Park, H. Lee, J. Park, and W. Lee, “The complete mitochondrial genome of Paracolopha morrisoni (Baker, 1919) (Hemiptera: Aphididae)," Mitochondrial DNA Part B., vol. 4, no. 2, pp. 3037-3039, 2019.

[66] B. Y. Seo, J. Park, W. Kwon, and J. Park, "The complete mitochondrial genome of Aiolocaria hexaspilota (Hope, 1831)(Coleoptera: Coccinellidae)," Mitochondrial DNA Part B., vol. 4, no. 1, pp. 1472-1474, 2019.

[67] J. Park, W. Kwon, B. Zhu et al., "Complete mitochondrial genome sequence of an aflatoxin B and G producing fungus, Aspergillus parasiticus," Mitochondrial DNA Part B, vol. 4, no. 1, pp. 947-948, 2019.

[68] J. Park and J. Park, "Complete mitochondrial genome of the gate-keeper ant Colobopsis nipponica (Wheeler, W.M., 1928) (Formicidae: Hymenoptera)," Mitochondrial DNA Part B., vol. 6, no. 1, pp. 86-88, 2021.

[69] H. Lee, J. Park, J. Lee, K.-J. Hong, J. Park, and W. Lee, "The complete mitochondrial genome of Ceutorhynchus obstrictus (Marsham, 1802)(Coleoptera: Curculionidae)," Mitochondrial DNA Part B., vol. 4, no. 2, pp. 3096-3098, 2019.

[70] J. Park, W. Kwon, and J. Park, "The complete mitochondrial genome of Ectomomyrmex javanus Mayr, 1867 (Hymenop- tera: Formicidae)," Mitochondrial DNA Part B., vol. 4, no. 1, pp. 1636-1637, 2019.

[71] J. Park, Y. Kim, and H. Xi, "The complete mitochondrial genome sequence of Chinese minnow in Korea, Rhynchocypris oxycephalus (Sauvage and Dabry de Thiersant, 1874)," Mitochondrial DNA Part B., vol. 4, no. 1, pp. 662-663, 2019.

[72] J. Park, C.-H. Park, and J. Park, "Complete mitochondrial genome of the $\mathrm{H} 3$ haplotype Argentine ant Linepithema humile (Mayr, 1868) (Formicidae; Hymenoptera)," Mitochondrial DNA Part B., vol. 6, no. 3, pp. 786-788, 2021.

[73] J. Park and J. Park, "Complete mitochondrial genome of the jet ant Lasius spathepus Wheeler, WM, 1910 (Formicidae; Hymenoptera)," Mitochondrial DNA Part B., vol. 6, no. 2, pp. 505-507, 2021.

[74] J. Park, H. Xi, and J. Park, "Complete mitochondrial genome of the acrobat ant Crematogaster teranishii Santschi, 1930 (Formicidae; Hymenoptera)," Mitochondrial DNA Part B., vol. 6, no. 2, pp. 593-595, 2021.

[75] K.-J. Hong, W. Ki, D.-S. Park et al., "The complete mitochondrial genome of Alphitobius diaperinus Panzer, 1797 (Coleoptera: Tenebrionidae)," Mitochondrial DNA Part B., vol. 5, no. 3, pp. 2291-2293, 2020.

[76] K.-J. Hong, W. Ki, H. Lee, J. Park, and W. Lee, “The second complete mitochondrial genome of Alphitobius diaperinus Panzer, 1797 (Coleoptera: Tenebrionidae): investigation of intraspecific variations on mitochondrial genome," Mitochondrial DNA Part B., vol. 5, no. 3, pp. 2979-2981, 2020.

[77] H.-J. Kim, J.-Y. Hwang, K.-J. Park et al., “The first complete mitogenome of Cervus canadensis nannodes (Merriam, 1905)," Mitochondrial DNA Part B., vol. 5, no. 3, pp. 22942296, 2020.

[78] J. Park, H. Xi, and J. Park, "The complete mitochondrial genome of Rotunda rotundapex (Miyata \& Kishida, 1990) (Lepidoptera: Bombycidae)," Mitochondrial DNA Part B., vol. 5, no. 1, pp. 355-357, 2020.

[79] J. Park, Y. Kim, W. Kwon, H. Xi, and J. Park, "The complete mitochondrial genome of Neocaridina heteropoda koreana Kubo, 1938 (Decapoda: Atyidae)," Mitochondrial DNA Part B., vol. 4, no. 2, pp. 2332-2334, 2019.

[80] B. Y. Seo, J. Cho, G.-S. Lee, J. Park, and J. Park, “The complete mitochondrial genome of Exorista japonica (Townsend, 1909)(Diptera: Tachinidae)," Mitochondrial DNA Part B., vol. 4, no. 2, pp. 2244-2245, 2019.

[81] J. Park, H. Xi, and C. Park, "The complete mitochondrial genomes from three body color variants of sea cucumbers, Apostichopus japonicus (Selenka, 1867)," Mitochondrial DNA Part B., vol. 4, no. 1, pp. 836-837, 2019.

[82] S. S. Choi, V. A. Bakalin, W. Kwon, and J. Park, "The complete mitochondrial genome of Douinia plicata (Lindb.) Konstant. et. Vilnet (Scapaniaceae, Jungermanniales)," Mitochondrial DNA Part B., vol. 6, no. 3, pp. 789-791, 2021.

[83] S. S. Choi, J. Min, W. Kwon, and J. Park, "The complete mitochondrial genome of Scapania ampliata Steph., 1897 (Scapaniaceae, Jungermanniales)," Mitochondrial DNA Part B., vol. 6, no. 2, pp. 686-688, 2021.

[84] J. Park, J. Lee, and W. Lee, "The complete mitochondrial genome of Aphis gossypii Glover, 1877 (Hemiptera: Aphididae) isolated from Leonurus japonicus in Korea," Mitochondrial DNA Part B., vol. 6, no. 1, pp. 62-65, 2021.

[85] Y. J. Jung, J. Jo, Y. Bae, H. Xi, M.-A. Seol, S.-H. Yoo et al., “The complete mitochondrial genome of Myzus persicae (Sulzer, 
1776; Hemiptera: Aphididae) isolated in Korea," Mitochondrial DNA Part B., vol. 6, no. 1, pp. 10-12, 2021.

[86] B. Kim, Y. Bae, J. Lee, J. Park, Y.-S. Choi, H. Ryu et al., “The complete mitochondrial genome of the far Eastern myotis: Myotis bombinus Thomas, 1906 in mainland of Korea (Chiroptera, Vespertilionidae)," Mitochondrial DNA Part B., vol. 6, no. 2, pp. 615-616, 2021.

[87] J. Park, W. Kwon, S.-B. Hong, and K.-H. Han, "First record of the complete mitochondrial genome of a saprotrophic and opportunistic human pathogenic Fungus, Scopulariopsis brevicaulis," Han'guk Kyunhakhoe chi, vol. 48, no. 6, pp. 528531, 2020.

[88] S. Joo, J. Lee, D.-Y. Lee, H. Xi, and J. Park, "The complete mitochondrial genome of the millipede Epanerchodus koreanus Verhoeff, 1937 collected in limestone cave of Korea (Polydesmidae: Polydesmida)," Mitochondrial DNA Part B., vol. 5, no. 4, pp. 3845-3847, 2020.

[89] H.-J. Kim, J.-Y. Hwang, K.-J. Park, H.-C. Park, H.-E. Kang, J. Park et al., "The complete mitochondrial genome of Cervus canadensis (Erxleben, 1777), as a model species of Chronic Wasting Disease (CWD)," Mitochondrial DNA Part B., vol. 5, no. 3, pp. 2621-2623, 2020.

[90] J. Park, J. Park, J.-H. Kim, J. R. Cho, Y. Kim, and B. Y. Seo, "The complete mitochondrial genome of Micromus angulatus (Stephens, 1836) (Neuroptera: Hemerobiidae)," Mitochondrial DNA Part B., vol. 4, no. 1, pp. 1467-1469, 2019.

[91] T. Han, H. Park, J.-H. Lee et al., "The complete mitochondrial genome of the subterranean termite, Reticulitermes kanmonensis Takematsu, 1999 (Isoptera: Rhinotermitidae)," Mitochondrial DNA Part B., vol. 2, no. 2, pp. 508-509, 2017.

[92] S. F. Altschul, T. L. Madden, A. A. Schäffer, J. Zhang, Z. Zhang, W. Miller et al., "Gapped BLAST and PSI-BLAST: a new generation of protein database search programs," Nucleic Acids Research, vol. 25, no. 17, pp. 3389-3402, 1997.

[93] P. Schattner, A. N. Brooks, and T. M. Lowe, "The tRNAscanSE, snoscan and snoGPS web servers for the detection of tRNAs and snoRNAs. Nucleic acids research," vol. 33, no. 2, pp. W686-W6W9, 2005.

[94] K. Katoh and D. M. Standley, "MAFFT multiple sequence alignment software version 7: improvements in performance and usability," Molecular Biology and Evolution, vol. 30, no. 4, pp. 772-780, 2013.

[95] J. Chen, Z. Hao, H. Xu et al., "The complete chloroplast genome sequence of the relict woody plant Metasequoia glyptostroboides Hu et Cheng," Frontiers in Plant Science, vol. 6, p. $447,2015$.

[96] N. Shukla, H. Kuntal, A. Shanker, and S. N. Sharma, "Mining and analysis of simple sequence repeats in the chloroplast genomes of genus Vigna," Biotechnology Research and Innovation., vol. 2, no. 1, pp. 9-18, 2018.

[97] W. Li, C. Zhang, X. Guo, Q. Liu, and K. Wang, "Complete chloroplast genome of Camellia japonica genome structures, comparative and phylogenetic analysis," PLoS One, vol. 14, no. 5, article e0216645, 2019.

[98] J.-H. Jeon and S.-C. Kim, "Comparative analysis of the complete Chloroplast Genome sequences of three closely related East-Asian wild roses (Rosa sect. Synstylae; Rosaceae)," Genes, vol. 10, no. 1, p. 23, 2019.

[99] J. Cheng, Z. Zhao, B. Li et al., "A comprehensive characterization of simple sequence repeats in pepper genomes provides valuable resources for marker development in Capsicum," Scientific Reports, vol. 6, no. 1, p. 18919, 2016.

[100] Y. Kim, H. Xi, and J. Park, “The complete chloroplast genome of Prince Ginseng, Pseudostellaria heterophylla (Miq.) Pax (Caryophyllaceae)," Mitochondrial DNA Part B., vol. 4, no. 2, pp. 2251-2253, 2019.

[101] S. G. Gandhi, P. Awasthi, and Y. S. Bedi, "Analysis of SSR dynamics in chloroplast genomes of Brassicaceae family," Bioinformation, vol. 5, no. 1, p. 16, 2010.

[102] Y. Kim, J. Park, and Y. Chung, "Comparative Analysis of Chloroplast Genome of Dysphania ambrosioides (L.) Mosyakin \& Clemants Understanding Phylogenetic Relationship in Genus Dysphania R," Br. Korean J Plant Res, vol. 32, no. 6, pp. 644-668, 2019.

[103] J. Park, H. Xi, and Y. Kim, "The complete chloroplast genome of Arabidopsis thaliana isolated in Korea (Brassicaceae): an investigation of intraspecific variations of the chloroplast genome of Korean A. Thaliana," International journal of genomics, vol. 2020, 18 pages, 2020.

[104] J. Park, J. Min, Y. Kim, and Y. Chung, "The comparative analyses of six complete chloroplast genomes of morphologically diverse Chenopodium album L. (Amaranthaceae) collected in Korea," International Journal of Genomics, vol. 2021, 15 pages, 2021.

[105] J. Park and H. Xi, "Genome Archive (R): standardized genome repository for supporting large-scale genome analyses," in Plant and Animal Genome XXVI Conference (January 13-17, 2018), PAG, 2018.

[106] D. Darriba, G. L. Taboada, R. Doallo, and D. Posada, “jModelTest 2: more models, new heuristics and parallel computing," Nature Methods, vol. 9, no. 8, p. 772, 2012.

[107] S. Kumar, G. Stecher, M. Li, C. Knyaz, and K. Tamura, "MEGA X: Molecular evolutionary genetics analysis across computing platforms," Molecular Biology and Evolution, vol. 35, no. 6, pp. 1547-1549, 2018.

[108] J. P. Huelsenbeck and F. Ronquist, "MRBAYES: Bayesian inference of phylogenetic trees," Bioinformatics, vol. 17, no. 8, pp. 754-755, 2001.

[109] C. L. Stone, R. D. Frederick, P. W. Tooley, D. G. Luster, B. Campos, R. A. Winegar et al., "Annotation and analysis of the mitochondrial genome of Coniothyrium glycines, causal agent of red leaf blotch of soybean, reveals an abundance of homing endonucleases," PLoS One, vol. 13, no. 11, article e0207062, 2018.

[110] X. Wang, L. Jia, M. Wang et al., "The complete mitochondrial genome of medicinal fungus Taiwanofungus camphoratus reveals gene rearrangements and intron dynamics of Polyporales," Scientific Reports, vol. 10, no. 1, pp. 16500-16514, 2020.

[111] C. Chen, Q. Li, R. Fu et al., "Characterization of the mitochondrial genome of the pathogenic fungus Scytalidium auriculariicola (Leotiomycetes) and insights into its phylogenetics," Scientific Reports, vol. 9, no. 1, p. 17447, 2019.

[112] A. Zubaer, A. Wai, and G. Hausner, "The mitochondrial genome of Endoconidiophora resinifera is intron rich," Scientific Reports, vol. 8, no. 1, p. 17591, 2018.

[113] D. V. Lavrov and W. Pett, “Animal mitochondrial DNA as we do not know it: mt-genome organization and evolution in nonbilaterian lineages," Genome Biology and Evolution, vol. 8, no. 9, pp. 2896-2913, 2016. 
[114] L. Wang, S. Zhang, J. H. Li, and Y. J. Zhang, "Mitochondrial genome, comparative analysis and evolutionary insights into the entomopathogenic fungus Hirsutella thompsonii," Environmental Microbiology, vol. 20, no. 9, pp. 3393-3405, 2018.

[115] Z. Xu, L. Wu, S. Liu, Y. Chen, Y. Zhao, and G. Yang, "Structure characteristics of Aspergillus egyptiacus mitochondrial genome, an important fungus during the fermentation of dark tea," Mitochondrial DNA Part B., vol. 3, no. 2, pp. 1135-1136, 2018.

[116] V. Joardar, N. F. Abrams, J. Hostetler, P. J. Paukstelis, S. Pakala, S. B. Pakala et al., "Sequencing of mitochondrial genomes of nine Aspergillus and Penicillium species identifies mobile introns and accessory genes as main sources of genome size variability," BMC Genomics, vol. 13, no. 1, p. 698, 2012.

[117] J. Park, S. H. Lee, and J. H. Kim, "Complete Genome Sequence of the Endosymbiotic Bacterium "Candidatus Riesia pediculicola"," Microbiology Resource Announcements., vol. 10, no. 18, article e01181-20, 2021.

[118] G. Bryan, J. McNicoll, G. Ramsay, R. Meyer, and W. De Jong, "Polymorphic simple sequence repeat markers in chloroplast genomes of Solanaceous plants," Theoretical and Applied Genetics, vol. 99, no. 5, pp. 859-867, 1999.

[119] W. Powell, M. Morgante, R. McDevitt, G. Vendramin, and J. Rafalski, "Polymorphic simple sequence repeat regions in chloroplast genomes: applications to the population genetics of pines," Proceedings of the National Academy of Sciences, vol. 92, no. 17, pp. 7759-7763, 1995.

[120] D. Takahashi, S. Sakaguchi, Y. Isagi, and H. Setoguchi, "Comparative chloroplast genomics of series Sakawanum in genus Asarum (Aristolochiaceae) to develop single nucleotide polymorphisms (SNPs) and simple sequence repeat (SSR) markers," Journal of Forest Research, vol. 23, no. 6, pp. 387392, 2018.

[121] A. Ebadi, N. Ghaderi, and Y. Vafaee, "Genetic diversity of Iranian and some European grapes as revealed by nuclear and chloroplast microsatellite and SNP molecular markers," The Journal of Horticultural Science and Biotechnology., vol. 94, no. 5, pp. 599-610, 2019.

[122] G. Y. Siew, W. L. Ng, M. F. Salleh et al., "Assessment of the genetic variation of Malaysian durian varieties using intersimple sequence repeat markers and chloroplast DNA sequences," Pertanika Journal of Tropical Agricultural Science, vol. 41, no. 1, 2018.

[123] Y. Li, X.-D. Hu, R.-H. Yang et al., "Complete mitochondrial genome of the medicinal fungus Ophiocordyceps sinensis," Scientific Reports, vol. 5, no. 1, p. 13892, 2015.

[124] S. Zhang, X.-N. Wang, X.-L. Zhang, X.-Z. Liu, and Y.J. Zhang, "Complete mitochondrial genome of the endophytic fungus Pestalotiopsis fici: features and evolution," Applied Microbiology and Biotechnology, vol. 101, no. 4, pp. 1593-1604, 2017.

[125] T.-S. Kim, J. G. Booth, H. G. Gauch et al., "Simple sequence repeats in Neurospora crassa: distribution, polymorphism and evolutionary inference," BMC Genomics, vol. 9, no. 1, p. 31, 2008.

[126] T. P. Michael, S. Park, T.-S. Kim, J. Booth, A. Byer, Q. Sun et al., "Simple sequence repeats provide a substrate for phenotypic variation in the Neurospora crassa circadian clock," PLoS One, vol. 2, no. 8, article e795, 2007.
[127] T.-S. Kim, B. A. Logsdon, S. Park, J. G. Mezey, and K. Lee, "Quantitative trait loci for the circadian clock in Neurospora crassa," Genetics, vol. 177, no. 4, pp. 2335-2347, 2007.

[128] B. Brankovics, T. Kulik, J. Sawicki et al., "First steps towards mitochondrial pan-genomics: detailed analysis of Fusarium graminearum mitogenomes," PeerJ, vol. 6, article e5963, 2018.

[129] Q. Yan, X. Liu, and Y. Zhang, "Reanalysis of the mitochondrial genome of the nematophagous fungus Hirsutella rhossiliensis," Acta Microbiologica Sinica, vol. 1, no. 1, 2018.

[130] Y.-J. Zhang, H.-Y. Zhang, X.-Z. Liu, and S. Zhang, "Mitochondrial genome of the nematode endoparasitic fungus Hirsutella vermicola reveals a high level of synteny in the family Ophiocordycipitaceae," Applied Microbiology and Biotechnology, vol. 101, no. 8, pp. 3295-3304, 2017.

[131] Y.-J. Zhang, S. Zhang, and X.-Z. Liu, "The complete mitochondrial genome of the nematode endoparasitic fungus Hirsutella minnesotensis," Mitochondrial DNA Part A., vol. 27, no. 4, pp. 2693-2694, 2016.

[132] Y. Liu, D. Tang, G. Zhang, M. Zhang, Y. Wang, Y. Wang et al., "Complete mitochondrial genome of Tolypocladium sp. YFCC 1805002 isolated from Ophiocordyceps sinensis in Baima Snow Mountain, Southwestern China," Mitochondrial DNA Part B., vol. 5, no. 1, pp. 171-173, 2020.

[133] C. Zhang, Y. Dai, G. Wang, C. Wang, Y. Gao, W. Deng et al., "Mitogenome of Tolypocladium guangdongense," Applied Microbiology and Biotechnology, vol. 104, no. 21, pp. 92959308, 2020.

[134] H. Fangliang, L. Yongquan, and C. Xinai, "The complete mitochondrial genome of a medicinal fungus, Tolypocladium ophioglossoides," Mitochondrial DNA Part B, vol. 2, no. 1, pp. 95-96, 2017.

[135] S. Zhang and Y.-J. Zhang, "Complete mitogenome of the entomopathogenic fungus Tolypocladium cylindrosporum," Mitochondrial DNA Part B., vol. 5, no. 1, pp. 680-682, 2020.

[136] Y.-J. Zhang, X.-Q. Yang, S. Zhang, R. A. Humber, and J. Xu, "Genomic analyses reveal low mitochondrial and high nuclear diversity in the cyclosporin-producing fungus Tolypocladium inflatum," Applied Microbiology and Biotechnology, vol. 101, no. 23-24, pp. 8517-8531, 2017. 